\title{
The TPS Direct Transport: a new method for transporting deformations in the Size-and-shape Space.
}

\author{
Valerio Varano $^{1}$. Stefano Gabriele ${ }^{1}$ - Luciano Teresi ${ }^{2}$ - Ian Dryden ${ }^{3}$. \\ Paolo E. Puddu ${ }^{4}$. Concetta Torromeo ${ }^{4}$. Paolo Piras Pa $^{4,5}$
}

Received: date / Accepted: date

\begin{abstract}
Modern shape analysis allows the fine comparison of shape changes occurring between different objects. Very often the classic machineries of Generalized Procrustes Analysis and Principal Component Analysis are used in order to contrast the shape change occurring among configurations represented by homologous landmarks. However, if size and shape data are structured in different groups thus constituting different morphological trajectories, a data centering is needed if one wants to compare solely the deformation representing the trajectories. To do that, inter-individual variation must be filtered out. This maneuver is rarely applied in studies using simulated or real data. A geometrical procedure named Parallel Transport, that can be based on various connection types, is necessary to perform such kind of data centering. Usually, the Levi Civita connection is used for interpolation of curves in a Riemannian space. It can also be used to transport a deformation. We demonstrate that this procedure does not preserve some important characters of the deformation, even in the affine case. We propose a novel procedure called 'TPS Direct Transport' which is able to perfectly transport deformation in the affine case and to better approximate non affine deformation in comparison to existing tools. We recommend to center shape
\end{abstract}

\section{Valerio Varano}

E-mail: valerio.varano@uniroma3.it

1 Department of Architecture, Roma Tre University, Roma, Italy.

2 Department of Mathematics \& Physics, Roma Tre University, Roma, Italy.

3 School of Mathematical Sciences, University of Nottingham, Nottingham, UK.

${ }^{4}$ Department of Cardiovascular, Respiratory, Nephrological and Geriatric Sciences, Sapienza University, Rome, Italy.

5 Department of Structural Engineering and Geotechnics, Sapienza University, Roma, Italy. data using the methods described here when the differences in deformation rather than in shape are under study.

Keywords Geometric Morphometrics - Shape Analysis - Inter-individual difference · Riemannian Manifold . Deformation cycle · Parallel Transport · Trajectory Analysis · Thin Plate Spline.

\section{Introduction}

\subsection{State of the Art}

Shape theory became central in many fields from computer vision to biological and medical applications; according to [41], it can be split in at least two principal frameworks: i) shape optimization, that is, the search for the best shape according to a criterion; examples include image segmentation and object tracking; and ii) shape analysis, consisting in the study of families of shapes for statistics, (automatic) cataloging, probabilistic modeling, etc. The former is more used in computer vision, and relies on continuum formulations (diffeomorphisms, active contours, parametrized surfaces etc.); the latter is more used in biological applications, and is based on the discrete sampling of shapes by means of homologous landmarks, which are carefully selected according to anatomical features. Recently, the field of computational anatomy translated the continuum approaches in medicine and biology.

In both frameworks, a shape is represented by a point on a Riemannian manifold, whose geodesic play a key role: geodesics are the natural paths along which to transport shapes, thus providing the essential tool to compare two different shapes or to interpolate between them. The essence of a Riemannian manifold is 
embodied in two notions: i) the metric, measuring the distance between points (that is, shapes); ii) the connection, yielding the geodesic connecting two points. It is worth noting that a connection has to be compatible with the metric, and can be built upon a Parallel Transport (PT), a rule for transporting vectors along geodesics.

Moreover, a PT is compatible with the metric when it does not change the length of vectors and the angles between them. For a given a metric, there exists a unique PT compatible with it, and having vanishing torsion: the Levi Civita (LC) PT. (The torsion of the connection is a slightly technical notion that we will discuss in the following).

According to [48], PT can be used in Shape theory for many purposes, such as defining geodesics between shapes, transporting a deformation from one shape to another, creating random sampling for statistical shape models. In the case of shapes sampled by homologous landmarks, the LC connection is often used to define the geodesic between two shapes, and to transport deformations [19]. However, it can be proved [47] that this approach does not preserve some important features of the deformation. [44] discusses the problem of comparing the deformations occurring between pairs of shapes and groups of shapes (in particular they formalize the concept of correspondence). The LV PT in the Kendall's Shape Space has been used in the past for 2D configurations $[14,18]$ with explicit formulas; recently, [47] proposed a new 2D explicit formula for LC PT in the Kendall's Size-and-Shape Space.

Many efforts have been done in recent years in order to unify shape metrics with deformation metrics, see $[7,27,28,34,48]$, whose complete review is far beyond the scope of the present paper. In particular, new metrics have been proposed together with the corresponding connections.

A morphometric approach based on diffeomorphisms (namely 'diffeomorphometry') has been recently patented for surfaces (not necessarily identified by homologous landmarks) acquired by CT scan [29]. For example, [42](fig.1) proposed a strategy that is similar in aim to the one presented here. $[28,31]$ revise the theory behind this attractive approach that traces back to [46] and [45]. It stems from the computation of geodesics by means of the Large Diffeomorphic Deformation Metric Mapping (LDDMM) approach for which we refer the reader to $[13,28]$ and references therein.

In classic Geometric Morphometrics, [2], [9], the deformation between two shapes is evaluated using the Thin Plate Spline (TPS) method, that is a function that minimizes the bending energy [1].
TPS, however, is a non-bijective interpolating function, and has no inherent restrictions to prevent folding, even if some efforts have been made to tackle this issue [11]. Moreover, given two shapes, a target and a source, TPS is 'source dependent' and swapping target with source yields a different deformation path. In Geometric Morphometrics it is a consolidated practice to decompose the whole deformation in affine and non affine components. The same is done in other contexts such as in the "deformotion" approach proposed by [49]. [41] proposed a tri-partite decomposition of the deformation (translation, scaling, all the rest) in the framework of the active contour approach, while [32] did the same in the context of LDDMM, in order to manage the non scale-invariance of LDDMM transformations. None of the aforementioned methods yield a PT compatible with the affine/non affine decomposition and do not consider the PT of the affine part of the deformation toward a target shape. When a $\mathrm{PT}$ is built upon the above mentioned methods, the LC connection is the main tool for transporting deformation. This PT, based on the LC connection, is dependent from the path, and some efforts have been done to propose path-independent strategies $[12,25]$.

Table 1 (far to be exhaustive) summarizes the principal features of the most common approaches proposed in the last years.

In the present paper we propose a PT that has two properties: i) it is independent from the path; ii) it is compatible with the affine/non affine decomposition. We couple this strategy with a data-centering aimed at eliminating inter-individual differences and with a trajectory analysis aimed at recovering the original deformational series, once shapes have been transported. In the present article we focus on the landmark-based shape theory, i.e. Geometric Morphometrics.

Geometric Morphometrics begins with the seminal contributions of Kendall [15, 16]. He proposed a criterion to eliminate all non shape-informed differences to evaluate dissimilarities between shapes. In the case of shapes sampled by homologous landmarks, the non shape-informed attributes are size, translation and rotation. Kendall [16] showed how shapes can be represented as points on a Riemannian manifold of dimension $m \times(k-1)-1-m(m-1) / 2$, were $m$ is the dimension of the ambient space, and $k$ the number of landmarks. This manifold is named Shape Space. The pole of the Shape Space is usually taken at the average of all configurations, called consensus. When the variation around the consensus is small, the geodesic Procrustes Distance is approximated by its projection (usually orthogonal) on the tangent space to the consensus. The aligned coordinates are then often subjected to ordina- 
Table 1 Comparison of PT approaches. L/R= Left/Right; C/S = Cartan-Schouten

\begin{tabular}{|c|c|c|c|c|c|c|c|c|}
\hline Methods & $\begin{array}{l}\text { Space } \\
\text { dimension }\end{array}$ & $\mathrm{PT}$ & Torsion & Curvature & $\begin{array}{l}\text { Scale } \\
\text { invariant }\end{array}$ & $\begin{array}{l}\text { Affine } \\
\text { preserv. }\end{array}$ & $\begin{array}{l}\mathrm{PT} \\
\text { calc. }\end{array}$ & $\begin{array}{l}\text { Relevant. } \\
\text { Ref. }\end{array}$ \\
\hline Kendall Size \& Shape & $(k-1) m$ & $\mathrm{LC}$ & 0 & $\neq 0$ & $\mathrm{NO}$ & $\mathrm{NO}$ & Algebraic & {$[14,18,20,47$} \\
\hline LDDMM & $\infty$ & $\mathrm{LC}$ & 0 & $\neq 0$ & $\mathrm{NO}$ & $\mathrm{NO}$ & Num. Integr. & $\begin{array}{l}{[5,27,28,30]} \\
{[43,46]}\end{array}$ \\
\hline Mod. LDDMM & $\infty$ & $\mathrm{LC}$ & 0 & $\neq 0$ & YES & $\mathrm{NO}$ & Num. Integr. & {$[32]$} \\
\hline Stat. Vel. Field & $\infty$ & symm. C/S & 0 & $\neq 0$ & YES & $\mathrm{NO}$ & Algebraic. & {$[22,23]$} \\
\hline Stat. Vel. Field & $\infty$ & $\mathrm{L} / \mathrm{R} \mathrm{C} / \mathrm{S}$ & $\neq 0$ & 0 & YES & $\mathrm{NO}$ & Algebraic. & {$[22,23]$} \\
\hline Active Contour & $\infty$ & $\mathrm{LC}$ & 0 & $\neq 0$ & NO & $\mathrm{NO}$ & Num. Integr. & {$[40]$} \\
\hline Mod. Active Contour & $\infty$ & $\mathrm{LC}$ & 0 & $\neq 0$ & YES & $\mathrm{NO}$ & Num. Integr. & {$[41]$} \\
\hline Parametrized surfaces & $\infty$ & $\mathrm{LC}$ & 0 & $\neq 0$ & $\mathrm{NO}$ & $\mathrm{NO}$ & Num. Integr. & {$[48]$} \\
\hline TPS Space & $(k-1) m$ & DT & $\neq 0$ & 0 & YES & YES & Algebraic & This study \\
\hline
\end{tabular}

tion methods, such as Principal Component Analysis (PCA) for further analysis. If one is interested also in the size variation, the appropriate space is the Size-andshape Space, where only translations and rotations are eliminated by obtaining the so called forms.

\subsection{The contributions of the present paper}

In this paper we deal with trajectory of forms, defined as ordered sequence of forms, and we investigate the differences among trajectories, irrespective of the differences of the forms they contain.

We show that shape analysis of trajectories should be performed only after a proper representation of each shape of a trajectory has been obtained, and before applying ordination methods. In fact, studying the form of a trajectory means studying how the deformation changes along each path irrespectively of the actual form to which these deformations apply. The independence of the deformation from the form to which it is applied is critical: it implies that any form variation between individuals at the beginning of each trajectories must be completely filtered out. Often, in statistics, inter-group differences are eliminated by applying a group-mean centering, optionally followed by the Grand Mean addition.

A problem arises if the data are shape or form data. Very frequently the LC connection on the Shape Space is used to compute the geodesics between two shapes $[18,20]$. Sometimes it is also used to transport a deformation along this geodesic, in order to apply a deformation from one shape to the another shape $[14,19,48]$, where the torsion of the connection is zero. Formally, this procedure could be applied in order to center data in the Shape Space, but it is revealed to be inadequate in some cases because it does not preserve the physical meaning of the deformation during the path.
Many efforts have been done in recent years in order to unify shape metrics with deformation metrics $[4,21,27,30,32,34,44,48]$; in general, independently from the used description (landmarks based, parametric, diffeomorphism based), new metrics have been proposed together with the corresponding induced LC connections. Because LC connection can be written in terms of the metric, PTs are determined uniquely by metric issues.

Here we show how a new connection that we call 'TPS Connection' allows, by means of a 'TPS DT', to compare different form trajectories by performing a data centering which maintains the nature of the deformations. In particular the DT is compatible with the decomposition of the deformation to affine and non affine components. The adjective 'Direct' means that PT does not depend on the path, then the Riemannian curvature of the connection is zero. Moreover the DT is compatible with an introduced new Riemannian metric (TPS metric) but is different from the LC transport, as the torsion of the connection is different from zero. Despite all the technicalities related to the landmarks based description, the idea to give up the symmetry of the connection to obtain a connection flat and compatible with a significant decomposition of the tangent spaces could be exported to other contexts.

In shape analysis flat connections are rarely used, but in classical differential geometry there are several flat connections which are compatible with a given metric. Left (respectively right) Cartan-Schouten connection is an example of a connection with absolute parallelism whose parallel transport has a closed form (the differential of the left (respectively right) translations) and which is compatible with any left (respectively right) invariant Riemannian metric. The DT which we propose here is a particular type of Weitzenbock' connection. A Weitzenbock connection is specified by a frame field everywhere (see e.g. [25]). 
We used simulated datasets and an a priori known set of affine and non affine parametrized deformations in order to build properly pre-processed form trajectories to be used in standard ordination methods such as PCA. As far as we know there are few or no contributions aimed at performing such "reverse engineering" strategy. This allows to control the properties of final result and to perform specific performance analyses allowing to appreciate if the original deformation trajectories are properly transported toward the target shape. In addition, we illustrate the methodology with an application in cardiology, which motivates the work.

To summarize, the main contributions of the present work are:

\section{- Trajectory Analysis via Data Centering in Riemannian Manifold}

While PCA on centered data is used in many papers (e.g. the concept of atlas in functional anatomy $[5,28])$, as well as trajectory analysis via the use of different kinds of PT ( $[10,24,33,36,50,51])$. However, the idea of performing a shape analysis on the shapes of trajectory themselves, evaluated at physiological homologous times in order to assess the biological function has been introduced the first time in [35] and formalized in [47]. In that papers, has been widely shown as this procedure leads to better disease classification.

\section{- TPS Metric}

In both LDDMM and active contour frameworks, it has been proposed a decomposition of the deformation (together with a compatible metric), [32] and [41]; this decomposition only uncouples scaling from the rest of the deformation, without distinguishing between global affine deformations (size, aspect ratio and shear), and local ones (non affine component). Such a recognition of the difference between these two features of a deformation is instead present in the original formulation of TPS by Bookstein, but the decomposition is not accompanied by a Riemannian metric, being the bending energy only a singular metric which vanishes on all the affine deformations.

\section{- TPS Direct Transport}

The main features of the DT are: 1) it is compatible with the above mentioned TPS metric, 2) it is compatible with the given decomposition, 3) it is path independent, i.e. it induces a flat space. In particular the first feature means that the DT is an isometry with respect to the TPS metric. The second feature means that the transported vector of the original affine component coincides with the affine component of the transported vector and the same holds for the non affine component. The third fea- ture makes the whole procedure very simple from a conceptual point of view and computationally very cheap as it does not require any integration procedure: no calculation of the geodesics, no calculation of the PT along geodesics, only a closed form expression.

Moreover, the peculiarity of the DT, with respect to the most common PT used in shape analysis is the way it is built. It is not defined in terms of a given covariant derivative (e.g. in terms of Christoffel symbols), by integrating ODEs. It is directly formulated in terms of a given rule, by checking that it respects some abstract requirements characterizing any PT that represents a connection on a manifold [8]. This procedure is common in classical differential geometry. In fact, as stated above, the DT is a type of Weitzenbock connection.

\section{- Reverse Engineering Experiments}

The performance of PT here proposed, together with the whole procedure of data centering, is assessed by means of shape analysis on ad hoc shape data: we generate sequences of shapes by using parametrized deformation; our goal is to recover the values of the parameters used to generate the data set. This approach is rarely found in related literature.

All the following examples and analyses were performed in $\mathrm{R}$ using the package 'deformetrics' available on github. It can be installed using the install_github() function in 'devtools' $\mathrm{R}$ package by typing the following command line:

install_github('deformetrics/deformetrics',local=FALSE).

\section{The geometrical structure of the shape space}

A body $\mathcal{B}$ is an open subset of the $m$-dimensional Euclidean ambient space $\mathcal{E}^{m}$; the positions $x \in \mathcal{E}^{m}$ of $k$ points, called landmarks, define a configuration of the body, which can be represented as a $k \times m$ matrix $X=\left(x_{1}, \ldots, x_{k}\right)^{T}$; we denote with $\mathcal{C}_{m}^{k}$ the Configuration Space, that is, the set of all possible configurations.

The Shape Space $\Sigma_{m}^{k}$ can be defined as the quotient of $\mathcal{C}_{m}^{k}$ under the action of the group $\mathcal{S}(m)$ of the Euclidean similarity transformations in $\mathcal{E}^{m} \cdot \mathcal{S}(m)$ can be decomposed in three subgroups: translations $\mathcal{T}(m)$; rotations $S O(m)$; homothety or dilatation $\mathcal{H}(m)$. The Shape Space can be conveniently generated by removing similarity transformations one by one; the first step is to remove location, translating each configuration in such a way that the centroid lies on the origin $o$ of the Euclidean space. This brings us to the Centered Configuration Space $\mathcal{C C}_{m}^{k}$. A centered configuration is then defined as a configuration whose centroid lies on the origin. 
The successive filtering can be done by removing rotations-thus obtaining the Size-and-Shape Space $S \Sigma_{m}^{k}$; eventually, by removing size, we obtain the Shape Space as $\Sigma_{m}^{k}=S \Sigma_{m}^{k} / \mathcal{H}(m)$ [9]. To summarize, we consider the following spaces:

$$
\begin{aligned}
& \mathcal{C}_{m}^{k} \text {, Configuration Space; } \\
& \mathcal{C} C_{m}^{k} \text {, Centered Configuration Space; } \\
& \mathcal{S} \Sigma_{m}^{k}=\mathcal{C} C_{m}^{k} / S O(m), \text { Size-and-Shape Space; } \\
& \Sigma_{m}^{k}=\mathcal{S} \Sigma_{m}^{k} / \mathcal{H}(m), \text { Shape Space; }
\end{aligned}
$$

For each of the aforementioned spaces a suitable parametrization is needed. Here the Centered Configuration Space $\mathcal{C C}_{m}^{k}$ is parametrized in two complementary ways: centered landmarks or Helmertized landmarks. The first is a redundant parametrization while the second is a strict one. Both the parametrizations are obtained by pre-multiplying the coordinates matrix $X$ by a suitable matrix.

We define the centered configuration $X_{C}$ as the $k \times m$ matrix:

$X_{C}=C X$

where $C=I_{k}-\frac{1}{k} 1_{k} 1_{k}^{T}, I_{k}$ is the $k \times k$ identity matrix and $1_{k}$ is a $k \times 1$ column of ones.

We define the Helmertized landmarks $X_{H}$ as the $(k-1) \times m$ matrix:

$X_{H}=H X$

where $H$ is the so called Helmert sub-matrix. The $j-t h$ row of the Helmert sub-matrix $H$ is given by

$$
\left(h_{j}, \ldots, h_{j},-j h_{j}, 0, \ldots, 0\right), \quad h_{j}=-(j(j+1))^{-1 / 2}
$$

and so the $j-t h$ row consists of $h_{j}$ repeated $j$ times, followed by $j h_{j}$ and then $k-j-1$ zeros, $j=1, \ldots, k-1$. One can switch from one parametrization to the other by using the properties:

$H^{T} H=C$,

and then

$X_{C}=H^{T} X_{H}, \quad X_{H}=H X_{C}$.

The form (otherwise called size-and-shape) of a configuration $X$ is the equivalence class $[X]_{S} \in S \Sigma_{m}^{k}$ represented by:

$[X]_{S}=\left\{X_{C} Q: Q \in S O_{m}\right\}$.

Finally, the shape of a configuration $X$ is the equivalence class $[X] \in \Sigma_{m}^{k}$ defined as:

$[X]=[X]_{S} /\left\|X_{C}\right\|$ where $\left\|X_{C}\right\|=\left(\operatorname{trace}\left(X_{C}^{T} X_{C}\right)\right)^{1 / 2}$ is the Centroid Size (CS) of $X_{C}$, the most used measure of size in Geometric Morphometrics. We call an icon a particular member of the shape set $[X]$ which is taken as being representative of the shape. To summarize, we consider the following elements:

Configuration: $\quad X \in \mathcal{C}_{m}^{k}$;

Centered Configuration: $X_{C}$, or $X_{H} \in \mathcal{C C}_{m}^{k}$;

Form or Size-and-Shape: $[X]_{S} \in \mathcal{S} \Sigma_{m}^{k}$;

Shape: $\quad[X] \in \Sigma_{m}^{k}$.

Let us note that the procedure described above defines implicitly an atlas for the shape space, which inherits a manifold structure. Actually, the Shape Space by Kendall has a richer geometric structure, being endowed with: i) a Riemannian structure, defined by a metric $\left(g_{\Sigma}\right)$ on the tangent bundle; ii) a distance $\left(d_{\Sigma}\right)$ on the manifold; iii) a connection, defined by a covariant derivative $\left(\nabla_{\Sigma}\right)$ on the tangent bundle. It is important to stress that, in principle, these definitions are independent of each other, and the richness of the resulting geometric structure is overshadowed by both the elegance of the Kendall's construction, and by the tacit identification $\mathcal{C C}_{m}^{k} \equiv \mathbb{R}^{(k-1) m} \equiv \mathcal{E}^{(k-1) m}$ of the centered configuration space $\mathcal{C C}_{m}^{k}$ with the $(k-1) \times m$ Euclidean Space $\mathcal{E}^{(k-1) m}$; in particular, it is assumed that this identification holds for each level of the geometrical structure. The meaning and the consequences of this assumption will be discussed below.

Once accepted that the entire geometrical structure of $\mathcal{E}^{(k-1) m}$ is inherited by $\mathcal{C C}_{m}^{k}$, one observes that the regular part of the shape space $\Sigma_{m}^{k}$ is built by a sequence of Riemannian isometric maps: a quotient map $\pi$ (submersion) followed by an hortogonal projection $\varpi$ (immersion):

$\mathcal{C} \mathcal{C}_{m}^{k} \equiv \mathcal{E}^{(k-1) m} \underset{\text { submersion }}{\stackrel{\text { quotient } \pi}{\longrightarrow}} S \Sigma_{m}^{k} \underset{\text { immersion }}{\stackrel{\text { orthogonal projection } \varpi}{\longrightarrow}} \Sigma_{m}^{k}$.

This sequence induces isometrically all the geometric structure from the configuration space $\mathcal{C}_{m}^{k}$ to the shape space $\Sigma_{m}^{k}$; details can be found in [16], [20].

Here, it is useful to recall that in the Euclidean space $\mathcal{E}^{k m}$, the tangent spaces at any point can be identified with a global vector space $\mathbb{R}^{k m}$, i.e. the translation space of $\mathcal{E}^{k m}$. Thus, to each pair of points $(Y, X)$ there corresponds a vector $V=Y-X \in \mathbb{R}^{k m}$. Vectors belonging to $\mathbb{R}^{k m}$ are called deformation vectors; the Euclidean metric tensor corresponding to the dot product $U \cdot V=\operatorname{trace}\left(U^{T} V\right)$ is then naturally used to define an Euclidean distance:

$d(Y, X)=\|Y-X\|=\sqrt{(Y-X) \cdot(Y-X)}$.

Without entering into details (which will be given in the next section), here we complete the picture of the 
Euclidean space structure by recalling that the connection on $\mathcal{E}^{k m}$, in particular the LC connection, gives rise to parallel transports that are simple translations.

The induced distance on the size-and-shape space $S \Sigma_{m}^{k}$ is:

$$
\begin{aligned}
d_{S}\left([Y]_{S},[X]_{S}\right) & =\inf _{Q \in S O(m)} d(Y Q, X) \\
& =\inf _{Q \in S O(m)}\|Y Q-X\| .
\end{aligned}
$$

This definition allows us to give a procedure to align a configuration $Y$ onto a configuration $X$. In particular the aligned configuration $\hat{Y}$ is obtained by means of an optimal rotation $\hat{Q}$ minimizing the Euclidean distance $\|Y Q-X\|$.

$\hat{Y}=Y \hat{Q}, \quad \hat{Q}=\operatorname{argmin}_{Q \in S O(m)}\|Y Q-X\|$.

It is possible to prove that $\hat{Q}$ is the rotational component coming from a polar decomposition of $Y^{T} X$ (see [9]). That is, $Y^{T} X=\hat{Q} U$ with $U \in \operatorname{Sym}\left(\mathbb{R}^{m m}\right)$ and $\hat{Q} \in S O(m)$. It follows,

$$
\hat{Q}^{T} Y^{T} X=(Y \hat{Q})^{T} X=\hat{Y}^{T} X=U .
$$

As a consequence, we can say that two configurations $\hat{Y}, X$ are optimally aligned if and only if the matrix $\hat{Y}^{T} X$ is symmetric, i.e $\hat{Y}^{T} X \in \operatorname{Sym}\left(\mathbb{R}^{m m}\right)$. This pairwise alignment is called Ordinary Procrustes Analysis (OPA) without scaling. When one deals with several configurations $X_{i}$ a technique called Generalized Procrustes Analysis (GPA), allows, by means of an iterative algorithm, to define an average configuration $X_{G M}$, called the Grand Mean (GM), and, simultaneusy, to align every $X_{i}$ to $X_{G M}$. In other words, at the end of a GPA, one obtains a set of configurations $\hat{X}_{i}$, such that for each $i, \hat{X}_{i}^{T} X_{G M} \in \operatorname{Sym}\left(\mathbb{R}^{m m}\right)$

Each tangent space $T_{X}\left(\mathcal{C C}_{m}^{k}\right)$ at the centered configuration $X_{C} \in \mathcal{C C}_{m}^{k}$ can be identified with the global vector space $\mathbb{R}^{(k-1) m}$; moreover, with respect to the quotient map $\pi: \mathcal{C C}_{m}^{k} \rightarrow S \Sigma_{m}^{k}, T_{X}\left(\mathcal{C C}_{m}^{k}\right)$ splits into a vertical and a horizontal subspace:

$$
T_{X}\left(\mathcal{C C}_{m}^{k}\right)=\mathcal{V}_{X} \oplus \mathcal{H}_{X}
$$

characterized as follows:

$$
\begin{gathered}
\mathcal{V}_{X}=\left\{V \in \mathcal{T}_{X}\left(\mathcal{C C}^{k m}\right): V=X W, \text { with } W=-W^{T}\right\}, \\
\mathcal{H}_{X}=\left\{V \in \mathcal{T}_{X}\left(\mathcal{C C}^{k m}\right): \text { with } V^{T} X=\left(V^{T} X\right)^{T}\right\},
\end{gathered}
$$

In practice, a vertical vector at $X$ is an infinitesimal rotation of the configuration $X$, while a horizontal vector is a vector that, added to $X$, yields a configuration $Y=X+V$ aligned with $X$. Conversely, given a configuration $Y$ aligned with $X$, their difference is horizontal.
The key feature of the horizontal subspace $\mathcal{H}_{X}$ is that it is isometric to $\mathcal{T}_{[X]_{S}}\left(\mathcal{S} \Sigma_{m}^{k}\right)$, the tangent space of $S \Sigma_{m}^{k}$ at the form $[X]_{S}$. This feature allows us to represent vectors in $\mathcal{T}_{[X]_{S}}\left(\mathcal{S} \Sigma_{m}^{k}\right)$ through their corresponding vectors in $\mathcal{H}_{X}$, which are easier to handle.

\section{Deformation maps and Form trajectories}

One basic notion that will be crucial in the following is that of a deformation map, a smooth, interpolant map $\Phi: \mathcal{E}^{m} \rightarrow \mathcal{E}^{m}$. Given a pair of configurations $X, Y \in$ $\mathcal{C}_{m}^{k}$, we shall write

$$
Y=\Phi(X)
$$

to say that $Y$ is a deformation of $X$, that is

$y_{i}=\Phi\left(x_{i}\right), \quad \forall x_{i} \in X, y_{i} \in Y$.

Here $X$ is the source and $Y$ is the target. Note that the deformation acts on the whole space $\mathcal{E}^{m}$, rather than just on a set of landmarks. A bijective deformation $\Phi$ is a diffeomorphism from $\mathcal{E}^{m}$ to itself. Furthermore, note that the deformation is a notion pertaining to the Configuration Space rather than to the Shape Space or Size-and-Shape Space. A family of deformations $\Phi_{t}: \mathcal{E}^{m} \rightarrow \mathcal{E}^{m}$, smoothly parametrised by a scalar $t$, is called a motion. Given a motion $\Phi_{t}$, we define the discrete trajectory of the configuration $X$ under the action of $\Phi_{t}$ as the sequence:

$\mathcal{T}_{\Phi}(X)=\left(X_{t_{1}}, \ldots, X_{t_{n}}\right), \quad$ with $X_{t_{i}}=\Phi_{t_{i}}(X)$.

We shall tackle two main examples:

1. Different motions of the same body: given different motions $\Phi_{t}^{j}$, and a single configuration $X$, we can generate many different discrete trajectories:

$$
\mathcal{T}_{\Phi^{j}}(X)=\left(X_{t_{1}}^{j}, \ldots, X_{t_{n}}^{j}\right), \quad \text { with } X_{t_{i}}^{j}=\Phi_{t_{i}}^{j}(X) ;
$$

2. Same motion of different bodies: given a motion $\Phi_{t}$ and different configurations $X^{\ell}$, we generate many different discrete trajectories:

$$
\mathcal{T}_{\Phi}\left(X^{\ell}\right)=\left(X_{t_{1}}^{\ell}, \ldots, X_{t_{n}}^{\ell}\right), \quad \text { with } X_{t_{i}}^{\ell}=\Phi_{t_{i}}\left(X^{\ell}\right)
$$

Please, note that the apex in $X_{t_{i}}^{j}$ or $X_{t_{i}}^{\ell}$ can refer both to a motion $\Phi_{t}^{j}$, as in the first item, or to a configuration $X^{\ell}$, as in the second one.

The same notion of discrete trajectory applies also to a sequence of forms; thus, we define the trajectory of the form $[X]_{S}$ under the action of $\Phi_{t}$ as the sequence:

$\mathcal{F} \mathcal{T}_{\Phi}(X)=\left(\left[X_{t_{1}}\right]_{S}, \ldots,\left[X_{t_{n}}\right]_{S}\right), \quad$ with $X_{t_{i}}=\Phi_{t_{i}}(X)$ 
Our goal is the development of a procedure to compare forms' discrete trajectories, and be able to discriminate between intra- and inter-form variations.

If the displacements between the forms of a discrete trajectory are small enough, they can be considered as vectors belonging to a same tangent space of $\mathcal{S} \Sigma_{m}^{k}$; in this case form differences can be efficiently assessed by ordination analyses such as PCA performed on the covariance matrix. The problem arises when two or more forms' discrete trajectories span different and distant neighborhoods of the Size-and-Shape Space. In such a case, even if the deformations within each discrete trajectory are small, they cannot be compared: deformation vectors belong to very different tangent spaces. In differential geometry the tool for comparing vectors on different tangent spaces is Parallel Transport (PT) $[8,26,39]$.

\section{Parallel Transports and Riemannian Connections}

The PT on a manifold is related to the connection defined on its tangent bundle. To be more precise, according to [8], we begin by specifying the rule that any PT $\tau_{b, a}$ along a path from $a$ to $b$ has to fulfill:

$\tau_{b, a}: T_{a} \mathcal{M} \rightarrow T_{b} \mathcal{M}$, is linear, and non-singular.

$$
V_{a} \mapsto V_{b}
$$

moreover, for any point $c$ on the path

$\tau_{b, c} \circ \tau_{c, a}=\tau_{b, a}$

It follows from this that $\tau_{a, a}$ is the identity on $T_{a} \mathcal{M}$, and $\tau_{a, b}=\left(\tau_{b, a}\right)^{-1}$.

A parallel vector field is a vector field generated by parallel transporting a given vector along a path; thus, $W$ is a parallel field if $W_{b}=\tau_{b, a}\left(W_{a}\right)$ for each $b$ and some $a$ on the path. A connection is compatible with a metric $g$ if the PT is an isometry, that is

$g_{a}\left(V_{a}, W_{a}\right)=g_{b}\left(\tau_{b, a}\left(V_{a}\right), \tau_{b, a}\left(W_{a}\right)\right)$

for each pair of vector $V_{a}, W_{a}$, see [17].

Usually, a PT is defined by means of a covariant derivative $\nabla$ along a curve $\gamma$. A vector field $V$ is said to be parallel along $\gamma$ if:

$\nabla_{\dot{\gamma}} V=0$.

As shown in [39], the PT is usually defined in terms of the covariant derivative $\nabla$, but one can also reverse the process: assume a parallel transport $\tau$, and define the covariant derivative by a limit:

$\nabla_{V_{p}} U=\lim _{h \rightarrow 0} \frac{\tau_{h, 0}^{-1} U_{\gamma(h)}-U_{\gamma(0)}}{h}$
The torsion of the connection $\nabla$ is the tensor field:

$\nabla_{V} W-\nabla_{W} V-[V, W]$

with $[\cdot, \cdot]$ the Lie bracket. A connection is called symmetric when the torsion is null, for all $V, W$. A fundamental result of Riemannian Geometry is the existence of a unique symmetric connection compatible with the metric $g$, named the LC connection. The uniqueness of the LC connection allows us to transfer easily a connection from a Riemannian manifold to another one via isometric maps.

Since the work of [16], the LC connections on the Shape Space $\Sigma_{m}^{k}$ and on the Size-and-Shape Space $S \Sigma_{m}^{k}$ have been widely studied. As outlined in the previous Section, the regular part of the Shape Space can be defined by means of a sequence of Riemannian immersions and submersions starting from the Centered Configuration Space $\mathcal{C C}_{m}^{k}$, so that the LC connection on the Shape Space can be isometrically inherited from that on $\mathcal{C C}_{m}^{k}$ :

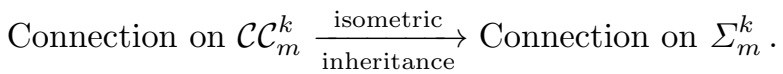

For $m=2$, PT has an explicit representation, while for $m=3$ PT can be evaluated by integrating the ordinary differential system (4.4). In both cases, the procedure has been used to interpolate curves on Shape Space [20], [18]. On the other hand, in [14] and [48] the LC parallel transport has been used to transfer a deformation from a shape to a different one. In [47] an explicit representation for $m=2$ has been introduced also for the Size-and-Shape Space, and used to compare form-discrete trajectories. In order to evaluate the utility of such a procedure, we need to better explain how the deformation can be defined and described.

\section{Describing Deformations: the Centered Thin Plate Spline}

In the previous sections we introduced two different notions of deformation: the deformation vector $V_{X}$, and the deformation map $\Phi$. Both are meant to transform a given configuration $X$ onto a deformed configuration $Y$ : we have $Y=X+V_{X}$ or, alternatively, $Y=\Phi(X)$. The differences between these two notions are:

- $V_{X}$ represents the displacements of the landmarks, while $\Phi$ is a map defined on the whole space.

- Given two close configurations $X$ and $Y$, the deformation vector from $X$ to $Y$ is unique, while there exist infinitely many maps $\Phi$ such that $\Phi(X)=Y$.

The choice of a suitable interpolation function $\Phi$ is not so obvious. A very known interpolating function is the 
Thin Plate Spline (TPS), introduced in [1] and developed in successive papers as $[3,37]$. The major drawback of the TPS is that it cannot prevent folding and cannot guaranty a diffeomorphism. For this reason it is not the mostly used regularization kernels in Computer Vision. Nevertheless it is the most used in Geometric Morphometrics, were the considered deformations are usually not so large to induce appreciable foldings. As stated above here we are considering the deformation occurring, within a single trajectory, between near configurations, then we will consider TPS as an acceptable representation.

The TPS representation of the deformation is often used due to the following advantages:

- The TPS interpolation has an explicit representation

- it decomposes the deformation into a global affine transformation and a set of local deformations which highlight changes at progressively smaller scales.

- it is based on the minimization of a cost-function, called the bending energy,

- the bending energy gauges the non-affine part of the deformation as a pseudo-distance between configurations.

The TPS representation allows us to obtain a very meaningful analysis of the deformation. On the other hand there are some drawbacks in the original formulation:

- The TPS is defined in the Configuration Space rather than in the Centered Configuration Space. This introduces an annoying term which represents a translation, even when two centered configurations are compared.

- The bending energy is a pseudo-distance, in fact it vanishes in the affine part of the deformation, so it cannot gauge the distance between two configuration related by a linear transformation (for example a simple shear).

- The bending energy is a pseudo-distance because it is not symmetric: the bending energy in deforming $X$ onto $Y$ is different from the one in deforming $Y$ onto $X$.

- The affine and non affine components, as coming from the TPS analysis, are not orthogonal in the Euclidean metric.

By following the notation of [9] we summarize the construction of the TPS, and we refer to $[1,9]$ for further details. In the Euclidean space $\mathcal{E}^{m}$, the $m$-tuple of interpolating TPS is a function $\Psi$ represented by the triple $(c, A, W)$, where: $c \in \mathcal{E}^{m}$ is a point represented by $(m \times 1)$ matrix; $A$ is a linear transformation of $\mathcal{E}^{m}$, represented by a $(m \times m)$ matrix; $W$ is a $(k \times m)$ matrix.
Given a point $x \in \mathcal{E}^{m}$, and a configuration $X \in \mathcal{C}_{m}^{k}$, we have

$y=\Psi(x)=c+A x+W^{T} s(x)$,

where $s(x)=\left(\sigma\left(x-x_{1}\right), \ldots, \sigma\left(x-x_{k}\right)\right)^{T}$ a is $(k \times 1)$ matrix, $x_{i} \in X$ is the position of the $\mathrm{i}$-th landmark, and

$$
\begin{aligned}
& \sigma(h)=\left\{\begin{array}{ll}
\|h\|^{2} \log (\|h\|) & \text { if }\|h\|>0 ; \\
0 & \text { if }\|h\|=0 .
\end{array} \quad \text { for } m=2\right. \\
& \sigma(h)=\left\{\begin{array}{ll}
-\|h\| & \text { if }\|h\|>0 ; \\
0 & \text { if }\|h\|=0 .
\end{array} \text { for } m=3\right.
\end{aligned}
$$

Given a source configuration $X$, and a target configuration $Y$, we can apply equation (5.6) landmark-wise, yielding to

$$
Y=1_{k} c^{T}+X A^{T}+S W, \quad \text { with } S_{i j}=\sigma\left(x_{i}-x_{j}\right) .
$$

There are $2 k$ interpolation constraints in equation (5.7), and we introduce $m \times(m+1)$ more constraints on $W$ in order to uncouple the affine and non affine parts:

$$
1_{k}^{T} W=0, \quad X^{T} W=0 .
$$

For a given pair $(X, Y)$ there exists a unique set of $m(1+m+k)=m+m^{2}+m k$ parameters for the triplet ( $c, A, W)$ that solve the problem (5.7), constrained with (5.8); the explicit solution can be found in the references.

Now we introduce some small changes to the original procedure in order to calculate everything directly in the Centered Configuration Space $\mathcal{C} \mathcal{C}_{m}^{k}$. By multiplying (5.7) by $C$, and exploiting the following properties of the operator $C$ :

$$
C=H^{T} H, \quad C 1_{k} c^{T}=0, \quad C W=W,
$$

(the last equation is a consequence of the constraint (5.8)) we can write

$Y_{H}=X_{H} A^{T}+S_{H} W_{H}$,

with $Y_{H}=H Y, X_{H}=H X$, and $W_{H}=H W(k-1) \times m$ matrices, and $S_{H}=H S H^{T}$ a $(k-1) \times(k-1)$ matrix. Everything is expresses in Helmertized coordinates that, as previously noted, is a strict parametrization of $\mathcal{C C}_{m}^{k}$. The constrained interpolation problem (5.7) can then be re-written as:

$\left[\begin{array}{c}Y_{H} \\ 0\end{array}\right]=\left[\begin{array}{cc}S_{H} & X_{H} \\ X_{H}^{T} & 0\end{array}\right]\left[\begin{array}{c}W_{H} \\ A^{T}\end{array}\right]$.

This linear system, provided that $S_{H}$ is invertible, yields the unique solution [9], [1]:

$$
\left[\begin{array}{c}
W_{H} \\
A^{T}
\end{array}\right]=\left[\begin{array}{cc}
S_{H} & X_{H} \\
X_{H}^{T} & 0
\end{array}\right]^{-1}\left[\begin{array}{c}
Y_{H} \\
0
\end{array}\right]=\left[\begin{array}{cc}
\Gamma_{11} & \Gamma_{21}^{T} \\
\Gamma_{21} & 0
\end{array}\right]\left[\begin{array}{c}
Y_{H} \\
0
\end{array}\right] .
$$


where

$\Gamma_{21}=\left(X_{H}^{T} S_{H}^{-1} X_{H}\right)^{-1} X_{H}^{T} S_{H}^{-1}$

$\Gamma_{11}=S_{H}^{-1}-S_{H}^{-1} X_{H} \Gamma_{21}$

are a $m \times(k-1)$ and a $(k-1) \times(k-1)$ matrices, respectively, which only depend on the source configuration $X$. Finally

$A^{T}=\Gamma_{21} Y_{H}, \quad W_{H}=\Gamma_{11} Y_{H}$,

so that the following decomposition holds:

$Y_{H}=X_{H} \Gamma_{21} Y_{H}+S_{H} \Gamma_{11} Y_{H}=X_{H} A^{T}+S_{H} W_{H}$.

The centered coordinates can be recovered simply by pre-multiplying with $H^{T}$. The quantity

$J(\Psi)=\nu \pi \operatorname{trace}\left(W_{H}^{T} S_{H} W_{H}\right)=\nu \pi \operatorname{trace}\left(Y_{H}^{T} \Gamma_{11} Y_{H}\right)$

(where $\nu=16$ for $m=2$ and is $\nu=8$ for $m=3$ (see appendix)) is called the bending energy. It can be proved that this corresponds to the integral:

$J(\Psi)=\sum_{i=1}^{n} \sum_{j=1}^{n} \sum_{k=1}^{n} \int_{\mathbb{R}^{m}}\left(\frac{\partial^{2} \Psi_{i}}{\partial x_{j} \partial x_{k}}\right)^{2}$

representing a mean elastic energy stored by the body as effect of the non-affine part of the deformation $\Psi[1]$. It is woth noting that in the past literature it has been always used, to the best of our knowledge, a different value for $\nu$ (e.g. in [3] eq.(5)). We provide, in appendix, the derivation of the right coefficient. The symmetric $(k-1) \times(k-1)$ matrix $\Gamma^{11}$ is named bending energy matrix.

It is important to note that the kernel of $\Gamma^{11}$ comprises the affine transformations of $X_{H}$ defined by $X_{H} \mapsto$ $X_{H} A$ :

$$
\Gamma_{11} X_{H} A=0, \forall X,
$$

and for all $m \times m$ matrices $A$. This property follows directly by the definition (5.9) that implies $\Gamma_{21} X_{H}=I$, and, once put in (5.10) implies $\Gamma_{11} X_{H}=0$.

\section{Gauging Deformations: The TPS Riemannian metric and the $\Gamma$ Energy}

In this section we will try to unify the two different notions of deformation introduced up to now, deformation vector and deformation map, and to overcome the main drawbacks of the original TPS tool.

This unification will be made by endowing the space $\mathcal{C C}_{m}^{k}$ with a new Riemannian structure based on the TPS. It is important to note that $\mathcal{C C}_{m}^{k}$ is a linear space, and any tangent space $T_{X}\left(\mathcal{C C}_{m}^{k}\right)$ at $X_{C}$ can be identified with the global vector space $\mathbb{R}^{(k-1) m}$; on the other hand, if a Riemannian metric is introduced, the aforementioned identification is not canonical, and depends on the chosen point. Consequently, $\mathcal{C C}_{m}^{k}$ would then be actually a linear space, but its structure not Euclidean; for example, geodesics may be different from straight lines, and parallel transports different from the identity (a typical example of this situation is the hyperbolic plane [48]).

In other words, if we take two centered configurations $X_{C}, Y_{C}$, we can always define their difference $V=Y_{C}-X_{C}$ with $V \in \mathbb{R}^{(k-1) m}$. But only if $X_{C}$ and $Y_{C}$ are near enough, does it makes sense to consider this difference as a vector belonging to the tangent space $T_{X_{C}}\left(\mathcal{C C}_{m}^{k}\right)$.

From now on, deformation vectors will have a subscript denoting the starting point, that is, the source configuration; moreover, we assume all the configurations to be centered, and represented by the Helmertized landmarks, and we shall drop the subscript ()$_{H}$; if no otherwise specified, each matrix is a $(k-1) \times m$ matrix.

Given a configuration $X$, and a deformation vector $V_{X} \in T_{X}\left(\mathcal{C C}_{m}^{k}\right)$, we may define a deformed configuration $Y$ by:

$Y=X+V_{X}$

According to the TPS decomposition, we can represent (6.15) by using (5.12):

$$
\begin{aligned}
Y & =X \Gamma_{21}\left(X+V_{X}\right)+S \Gamma_{11}\left(X+V_{X}\right) \\
& =X+X \Gamma_{21} V_{X}+S \Gamma_{11} V_{X} \\
& =X+X\left(A^{T}-I\right)+S W,
\end{aligned}
$$

Note $\Gamma_{21} X=I, \Gamma_{11} X=0$. It follows that, by means of TPS analysis, the deformation vector $V_{X}$ is decomposed into two summands:

$V_{X}=V_{X}^{\mathcal{U}}+V_{X}^{\mathcal{B}}$, with:

$V_{X}^{\mathcal{U}}=X \Gamma_{21} V_{X}=X\left(A^{T}-I\right)$, a uniform deformation of $X$;

$V_{X}^{\mathcal{B}}=S \Gamma_{11} V_{X}=S W$, a non-uniform deformation of $X$.

We note that in the following, as standard in GM, we will use the term uniform deformation as a synonymous of linear deformation. In fact, removing translations from affine deformations, we obtain linear deformations. Uniform means that the gradient of the deformation (the local strain) is constant. At the same time we will use non-uniform as a synonymous of non-linear.

In this way the notion of deformation map yields a useful decomposition of the deformation vector. It is important to note that $V_{X}^{\mathcal{U}}$ and $V_{X}^{\mathcal{B}}$ are not orthogonal 
with respect to the Euclidean metric, that is, in general, $\operatorname{trace}\left[\left(V_{X}^{\mathcal{U}}\right)^{T} V_{X}^{\mathcal{B}}\right] \neq 0$.

We can define a different metric $g_{X}$ at any $X$, such that $g_{X}\left(V_{X}^{\mathcal{U}}, V_{X}^{\mathcal{B}}\right)=0$; the metric $g_{X}$ can be naturally induced by the TPS parameter by defining the matrix $G_{X}$, such that:

$g_{X}(U, V)=\operatorname{trace}\left(U^{T} G_{X} V\right), \forall U, V \in T_{X}\left(\mathcal{C C}_{m}^{k}\right)$

The matrix $G_{X}$ can be decomposed into two symmetric summands, representing, respectively, the uniform metric and the bending metric:

$G_{X}=G_{\mathcal{U}}+G_{\mathcal{B}}$

Those two terms can be defined by using the TPS parameters $\Gamma_{21}$ and $\Gamma_{11}$, both depending on $X$, as follows:

$G_{\mathcal{U}}=\mu_{1} \Gamma_{21}^{T} \Gamma_{21}, \quad G_{\mathcal{B}}=\mu_{2} \Gamma_{11}$,

with $\mu_{1}$ and $\mu_{2}$ two positive scalars. It is easy to show that

$\operatorname{rank}\left(G_{\mathcal{U}}\right)=m, \quad \operatorname{rank}\left(G_{\mathcal{B}}\right)=(k-1)-m$,

and that the corresponding eigenspaces are linearly independent, so that $\operatorname{rank}(G)=k-1$, that is, full. Given $(6.16,6.17)$, the metric $g_{X}$ splits into two summands, $g_{X}=g_{\mathcal{U}}+g_{\mathcal{B}}$, and its action on vectors is rewritten as follows:

$$
\begin{aligned}
g_{X}(U, V) & =g_{\mathcal{U}}(U, V)+g_{\mathcal{B}}(U, V) \\
& =\mu_{1} \operatorname{trace}\left(U^{T} \Gamma_{21}^{T} \Gamma_{21} V\right)+\mu_{2} \operatorname{trace}\left(U^{T} \Gamma_{11} V\right)
\end{aligned}
$$

By means of the TPS metric tensor $g_{X}$, the tangent space $T_{X}$ can be decomposed as the direct sum

$T_{X}=\mathcal{U}_{X} \oplus_{g} \mathcal{B}_{X}$

where $\mathcal{U}_{X}$ is the subspace of uniform infinitesimal transformations of $X, \mathcal{B}_{X}$ is the subspace of those transformations that are infinitesimal pure bending of $X$ and $\oplus_{g}$ is the direct sum between subspaces that are orthogonal with respect to $g$.

Given the geometrical role of the TPS metric, one could ask what is the physical meaning of $g_{X}$. This can be made clear by evaluating separately $g_{\mathcal{U}}$ and $g_{B}$ on the pair $(V, V)$. The uniform part $g_{\mathcal{U}}$ gives:

$$
\begin{aligned}
g_{\mathcal{U}}(V, V) & =\mu_{1} \operatorname{trace}\left(V^{T} \Gamma_{21}^{T} \Gamma_{21} V\right) \\
& =\mu_{1} \operatorname{trace}\left(\left(\Gamma_{21} V\right)^{T} \Gamma_{21} V\right)
\end{aligned}
$$

By using (5.11) and (6.15) we obtain:

$g_{\mathcal{U}}(V, V)=\mu_{1} \operatorname{trace}\left((A-I)^{T}(A-I)\right)=\mu_{1}\|(A-I)\|^{2}$

where $\|$.$\| is the Frobenius norm of the space of m \times m$ matrices. Then, if we consider $\mu_{1}$ as an elastic stiffness,
$g_{\mathcal{U}}(V, V)$ is a quadratic elastic energy gauging the uniform deformation $(A-I)$. The non-uniform part $g_{\mathcal{B}}$ gives:

$g_{\mathcal{B}}(V, V)=\mu_{2} \operatorname{trace}\left(V^{T} \Gamma_{11} V\right)$

By using (6.15) and the property $\Gamma_{11} X=0$ we obtain:

$g_{\mathcal{B}}(V, V)=\mu_{2} \operatorname{trace}\left(Y^{T} \Gamma_{11} Y\right)=\mu_{2} J(\Phi)$

Then, $g_{\mathcal{B}}(V, V)$ is proportional to the previously introduced bending energy, and $\mu_{2}$ is an elastic bending stiffness. Finally, the value of $g_{X}(V, V)$ is called the $\Gamma$ energy associated with the deformation vector $V$. To be precise the values of $\mu_{1}$ and $\mu_{2}$ should depend on the elastic properties of the material of the considered body. However, as the two sub metrics act on orthogonal subspaces, the values of $\mu_{1}$ and $\mu_{2}$ will be immaterial in the present considerations concerning parallel transports.

\section{Transporting Deformations in the Centered Configuration Space: the TPS Direct Transport}

In the previous section we equipped the Centered Configuration Space $\mathcal{C C}_{M}^{k}$ with a new Riemannian metric: the TPS metric $g_{X}$. The obtained Riemannian space is a $(k-1) \times m$ dimensional linear space; following $(6.18)$ on each point the tangent space splits in a $m \times m$ dimensional subspace $\mathcal{U}_{X}$ of the uniform deformations and a $(k-1-m) \times m$ dimensional subspace $\mathcal{B}_{X}$ of the non-uniform deformations, mutually orthogonal with respect to the TPS metric. As previously seen, $\mathcal{U}_{X}$ can be parametrized by the linear space $M_{m \times m}$ of the $m \times m$ matrices. Let $\mathbf{e}_{i}(i, j=1 \ldots m)$ the standard orthonormal basis of $\mathcal{E}^{m}, \eta_{i j}=\mathbf{e}_{i} \otimes \mathbf{e}_{j}$ the standard basis of $M_{m \times m}$, we assume $\eta_{i j}^{\mathcal{U}}=X \eta_{i j}$ as a basis for $\mathcal{U}_{X}$.

The eigenvalue analysis of $\Gamma_{11}$ yields the principal warp eigenvectors $\gamma_{i}$, associated with the non vanishing eigenvalues $\lambda_{i},(i=1 \ldots(k-1-m))$. By construction $\gamma_{i}$ constitute an orthonormal basis with respect to the Euclidean metric. Any $V_{X}^{\mathcal{B}} \in \mathcal{B}_{X}$ can then be expressed as:

$$
\begin{aligned}
V_{X}^{\mathcal{B}}=S \Gamma_{11} S W & =\sum_{i=1}^{k-1-m} S \gamma_{i} \lambda_{i} \gamma_{i}^{T} S W \\
& =\sum_{i=1}^{k-1-m} \sum_{j=1}^{m} \eta_{i j}^{\mathcal{B}}\left(\eta_{i j}^{\mathcal{B}}\right)^{T} W
\end{aligned}
$$

where we introduce the principal warps, $\eta_{i j}^{\mathcal{B}}=\lambda_{i}^{\frac{1}{2}} S \gamma_{i} \otimes$ $\mathbf{e}_{j}$, a basis of $\mathcal{B}_{X}$ orthogonal (not orthonormal) with respect to the TPS metric. The corresponding components $\left(\eta_{i j}^{\mathcal{B}}\right)^{T} W$ are called partial warp scores.

The $(k-1) \times(k-1-m)$ matrix $E_{X}$, collecting all the principal warps of $X$ in columns is called the principal warps matrix and can be obtained as follows: 
- Perform a TPS analysis on $X$ and find $S$ and $\Gamma_{11}$,

- Perform an eigenvalue analysis on $\Gamma_{11}$ and obtain $\Gamma_{11}=\Gamma \Lambda \Gamma^{T}$ where $\Gamma$ is the $(k-1) \times(k-1)$ matrix containing, in column, the eigenvectors $\gamma_{i}$, and $\Lambda$ is the diagonal $(k-1) \times(k-1)$ matrix of the eigenvalues $\lambda_{1}, \ldots, \lambda_{k-1}$, ordered by increasing magnitude. The first $m$ eigenvalues will be equal to 0 ,

- Drop the first $m$ columns from $\Gamma$, by obtaining the $(k-1) \times(k-1-m)$ matrix $\bar{\Gamma}$, containing the principal warp eigenvectors by column,

- Drop the first $m$ rows and the first $m$ columns from $\Lambda$, by obtaining the $(k-1-m) \times(k-1-m)$ matrix $\bar{\Lambda}$,

- Define the $(k-1) \times(k-1-m)$ matrix $E_{X}=S \bar{\Gamma} \bar{\Lambda}^{1 / 2}$

Normalizing the bases of each subspace by using $\left(\mu_{1}, \mu_{2}, \lambda_{i}\right)$, we obtain, for the whole tangent space, the following orthonormal basis that we call standard basis:

$\eta_{i j}= \begin{cases}\frac{1}{\sqrt{\mu_{1}}} \eta_{i j}^{\mathcal{U}} & \text { if } 1 \leq i \leq m ; \\ \frac{1}{\lambda_{i} \sqrt{\mu_{2}}} \eta_{(i-m) j}^{\mathcal{B}} & \text { if } m+1 \leq i \leq k-1 .\end{cases}$

with $i=1 . . . k-1, j=1 . . m$.

After building the standard basis we can complete the introduced Riemannian structure by defining a parallel transport. A parallel transport can be defined by assigning a correspondence between the basis of the tangent spaces, but the choice of this correspondence is not canonical, and depends on our goal. The requirement that a connection be compatible with the metric determines univocally only the symmetric components of the connection. The symmetric part is unique, coincides with the LC connection, and the geodesic equations only depend on such components; but in general, the PT of a vector along a path is also affected by the skew symmetric components of the connection, which are proportional to the torsion [39].

Our goal is to probe the deformation between a pair (source, target) of configurations, and then apply the same deformation to a different source. The key point is in the definition of the notion of same deformation. We want to formalize this notion as an equivalence relation, i.e a binary relation reflexive, symmetric and transitive.

We propose the following definition: Two deformation vectors are equivalent if they can be described by the same TPS parameters. In particular, their uniform parts share the same linear transformation $A$. Concerning the non uniform parts, we recall that $W$ is a redundant representation of this part, its rank being determined by the constraint (5.8), i.e. it is different for each tangent space. Thus, our minimal requirement that two deformation vectors must fulfill in order to be equivalent, is that they store the same bending energy.
In terms of geometrical structure, the proposed equivalence between two vectors can be represented by a notion of parallelism determined by a PT that:

R.1 is compatible with the TPS metric,

R.2 is compatible with the decomposition (6.18),

R.3 preserves the uniform component,

R.4 is independent of the path.

Let $X_{a}$ be a source configurations, and $V_{a}, U_{b}$ two associated deformation vectors: The first item requires the PT to be an isometry with respect to the TPS metric.

$g_{a}\left(U_{a}, V_{a}\right)=g_{b}\left(\tau_{b, a}\left(U_{a}\right), \tau_{b, a}\left(V_{a}\right)\right)$

The second means:

$V_{b}^{\mathcal{U}}=\left(\tau_{b, a}\left(V_{a}\right)\right)^{\mathcal{U}}=\tau_{b, a}\left(V_{a}^{\mathcal{U}}\right)$

$V_{b}^{\mathcal{B}}=\left(\tau_{b, a}\left(V_{a}\right)\right)^{\mathcal{B}}=\tau_{b, a}\left(V_{a}^{\mathcal{B}}\right)$

The third requirement is illustrated by Fig. 1 in which it is clearly shown that the LC transports do not preserve the uniform component. The last requirement follows by the consideration that only a notion of absolute (global) parallelism can characterize an equivalence relation. In fact an absolute parallelism induces an equipollence relation [38]. In our construction two equipollent vectors represent the same deformation, applied to different starting configurations. In general, a relation between vectors, based on a path dependent connection, will not be reflexive, symmetric and transitive. For example, for a path dependent connection a vector is not parallel to itself if it is transported along a loop. In geometrical terms, independence of the path implies a vanishing Riemannian curvature and a non vanishing torsion. In the following, we propose a possible PT rule, compatible with the given requirements. In general, an absolute parallelism (also called a Weitzenboch connection) on a manifold can be built, when the manifold is parallelizable, by choosing a basis on each tangent space (the so-called Weitzenbock frame). Two vectors will be parallel if they have the same components on that basis. Furthermore, if the Weitzenbock frame, on each point of the manifold, is orthonormal with respect to a riemannian metric $g$, then the absolute parallelism will be compatible with $g$. Above we introduced, for each $X$, the orthonormal (with respect to TPS metric) standard basis $\eta_{i j}$. Starting from that basis it is possible to build any possible Weitzenbock frame $\mathcal{W}_{i j}$ by a suitable change of basis matrix $Q$ :

$\mathcal{W}_{i j}=Q_{p}^{i} \eta_{p j}$

with $i, p=1 . . . k-1, j=1 . . m$. The three requirements listed above restrict the possible choices of $Q$. In par- 
ticular the first requirement imply that $Q$ must be orthogonal (rotation or reflection):

R. $1 \leftrightarrow Q^{T} Q=I$,

The second requirement imply that $Q$ must be a block matrix:

$\mathrm{R} .2 \leftrightarrow Q=\left[\begin{array}{c|c}Q^{\mathcal{U}} & 0 \\ \hline 0 & Q^{\mathcal{B}}\end{array}\right]$

where $Q^{\mathcal{U}}$ is a $m \times m$ orthogonal matrix (i.e. $\left(Q^{\mathcal{U}}\right)^{T} Q^{\mathcal{U}}=$ $I)$, which rotates $\eta_{i j}^{\mathcal{U}}$ within $\mathcal{U}$ and $Q^{\mathcal{B}}$ is a $(k-1-m) \times$ $(k-1-m)$ orthogonal matrix $\left(\left(Q^{\mathcal{B}}\right)^{T} Q^{\mathcal{B}}=I\right)$, which rotates $\eta_{i j}^{\mathcal{B}}$ within $\mathcal{B}$. The third requirement set $Q^{\mathcal{U}}=I$

$\mathrm{R} .3 \leftrightarrow Q=\left[\begin{array}{c|c}I & 0 \\ \hline 0 & Q^{\mathcal{B}}\end{array}\right]$

While the above restrictions are important ingredients of the theory, the choice of $Q^{\mathcal{B}}$ (provided it is orthogonal) can depend on the applications.

In particular, for $Q^{\mathcal{B}}=I$, the proposed PT classifies two deformations as equivalent when they share the same uniform component, and the same, ordered partial-warp scores. Let us note that, given two different configurations $X_{a}$ and $X_{b}$, the $j-t h$ principal warp of $X_{a}$ may represent a deformation mode very different from the corresponding $j-t h$ warp of $X_{b}$. As principalwarps represent the standard basis of the subspace $\mathcal{B}_{X}$, we can introduce a criterion to rotate such a basis in order to obtain a more convenient correspondence between tangent spaces. One possible algorithm is:

- Assume a configuration $P$ as pole for the space;

- Assemble the $(k-1-m) \times(k-1)$ matrix of the principal warps for $P, \bar{\Gamma}_{P}$,

- For each configuration $X$, define $Q_{X}^{\mathcal{B}}$ as the rotational component of the polar decomposition of the $(k-1-m) \times(k-1-m)$ matrix $E_{P}^{T} E_{X}$.

For any $X$, this procedure minimizes the Euclidean distance $\left\|E_{X} Q_{X}^{\mathcal{B}}-E_{P}\right\|$ between the rotated principal warps of $X$, and the corresponding basis on the pole $P$. As a consequence, the corresponding non uniform deformation modes are made as similar as possible, albeit they will never coincide, being attached to different source configurations. Note that, in applications, the Pole can be conveniently chosen coincident with the Grand Mean of the considered dataset.

Once defined this Weitzenbock frame, we describe, in the following, how we can use it to transport a deformation from a point to another.

Let $X_{a}$ and $X_{b}$ be two source configurations, and $V_{a}, V_{b}$ the two associated deformation vectors, given by:

$V_{a}=X_{a}\left(A_{a}^{T}-I\right)+S_{a} W_{a}, \quad V_{b}=X_{b}\left(A_{b}^{T}-I\right)+S_{b} W_{b}$.
We say that $V_{b}$ is the parallel transport of a given $V_{a}$, that is, $V_{b}=\tau_{b, a}\left(V_{a}\right)$, if and only if the uniform part of $V_{b}$ equals that of $V_{a}$ :

$A_{b}=A_{a}$

and the non uniform part $W_{b}$ of $V_{b}$ solves the linear systems:

$$
X_{b}^{T} W_{b}=X_{a}^{T} W_{a}=0 \quad Q_{b}^{\mathcal{B}} E_{b}^{T} W_{b}=Q_{a}^{\mathcal{B}} E_{a}^{T} W_{a},
$$

The first equation of (7.20) constrains $W_{b}$ to be orthogonal to the affine part, while the second define the isometry in the subspace $\mathcal{B}$. This last requirement implies the conservation of the bending energy. The system (7.20) can be written as:

$$
\left[\begin{array}{c}
X_{b}^{T} \\
Q_{b}^{\mathcal{B}} E_{b}^{T}
\end{array}\right]\left[W_{b}\right]=\left[\begin{array}{c}
X_{a}^{T} \\
Q_{a}^{\mathcal{B}} E_{a}^{T}
\end{array}\right]\left[W_{a}\right] .
$$

The solution is given by

$\left[W_{b}\right]=\left[\begin{array}{c}X_{b}^{T} \\ Q_{b}^{\mathcal{B}} E_{b}^{T}\end{array}\right]^{-1}\left[\begin{array}{c}X_{a}^{T} \\ Q_{a}^{\mathcal{B}} E_{a}^{T}\end{array}\right]\left[W_{a}\right]$.

That can be re-written as:

$W_{b}=M_{b}^{-1} M_{a} W_{a}$

And so:

$V_{b}=\left(X_{b} \Gamma_{21 a}+S_{b} M_{b}^{-1} M_{a} \Gamma_{11 a}\right) V_{a}$,

where $\Gamma_{21 a}$ and $\Gamma_{11 a}$ are calculated assuming $X_{H}=X_{a}$ and $S_{H}=S_{a}$ in (5.9). The equation (7.21) characterizes $V_{b}$ as the parallel transport of $V_{a}$. It is immediate to verify that (7.21) is linear, invertible (for each pair of regular points $X_{a}$ and $X_{b}$ ) and independent from the path. It is also possible to prove that (7.21) is respectful of the general rule (4.2).

In fact, given a third point $X_{c}$, equation (7.21) writes:

$V_{c}=\tau_{c, a}\left(V_{a}\right)=\left(X_{c} \Gamma_{21 a}+S_{c} M_{c}^{-1} M_{a} \Gamma_{11 a}\right) V_{a} ;$

performing a successive PT toward $X_{b}$, one obtains

$V_{b}=\tau_{b, c}\left(V_{c}\right)=\left(X_{b} \Gamma_{21 c}+S_{b} M_{b}^{-1} M_{c} \Gamma_{11 c}\right) V_{c}$.

Inserting (7.22) in the last equation, using the properties $\Gamma_{21 c} V^{\mathcal{B}}=0, \forall V^{\mathcal{B}} \in \mathcal{B}_{X_{c}}$ and $\Gamma_{11 c} V^{\mathcal{U}}=0, \forall V^{\mathcal{U}} \in$ $\mathcal{U}_{X_{c}}$, and observing that, by construction, $X_{c} \Gamma_{21 a} V_{a} \in$ $\mathcal{U}_{X_{c}}$ and $S_{c} M_{c}^{-1} M_{a} \Gamma_{11 a} V_{a} \in \mathcal{B}_{X_{c}}$, one obtains:

$V_{\vec{F}}\left(X_{b} \Gamma_{21 c} X_{c} \Gamma_{21 a}+S_{b} M_{b}^{-1} M_{c} \Gamma_{11 c} S_{c} M_{c}^{-1} M_{a} \Gamma_{11 a}\right) V_{a}$ $=\left(X_{b} \Gamma_{21 a}+S_{b} M_{b}^{-1} M_{a} \Gamma_{11 a}\right) V_{a}=\tau_{b, a}\left(V_{a}\right)$,

where we used the properties $\Gamma_{21 c} X_{c}=I$, and $\Gamma_{11 c} V^{\mathcal{B}}=$ $S_{c}^{-1} V^{\mathcal{B}}, \forall V^{\mathcal{B}} \in \mathcal{B}_{X_{c}}$. 
Once checked that these abstract requirements are fulfilled by the DT, we can be sure that DT is, formally, a PT, inducing on the manifold a connection. In this work we are not interested in calculating the coefficients of this connection (i.e. the Christoffel symbols). This could be done by choosing a basis for vectors and using (4.5) to calculate covariant derivatives directly as limits [39] of a difference between near vectors. Because the DT does not depend on paths we don't need (in order to perform our data centering) to calculate geodesics and solve the differential equation (4.4). However, also without calculating coefficients of the connection, we are able to infer some important features of it directly from the PT: the connection is flat (zero Riemannian curvature) because the PT is independent from the path; the skew symmetric components of the connection (proportional the Torsion) will be different from zero, because the DT is different from the Levi Civita Connection.

We name the introduced connection the TPS connection and the related parallel transport TPS Direct Transport (because it is independent of the path). The Centered Configuration Space, equipped with the TPS metric and the TPS connection is named TPS Space.

\section{Transporting Deformations in the Size and Shape Space}

Once the Centered Configuration Space has been endowed with the TPS Riemannian structure, a further step is needed to complete the tools for comparing forms' discrete trajectories: we need to endow with such a structure to the Size-and-Shape Space.

As previously seen, the classical Size-and-Shape Space inherits by isometry the LC connection from the Centered Configuration Space, and because of the uniqueness of the LC connection, this inheritance is unique. In other words, once the (Euclidean) metric on the Centered Configuration Space is restricted to the Size-andShape Space, there exists only one connection compatible with such a metric, and torsion free (Levi-Civita). Given the uniqueness, it is then possible to build procedures, or explicit formulas, for parallel transporting vectors along paths, in particular along geodesic paths $[18,20,47]$.

As the torsion of the TPS connection is not null, it is not unique, and we cannot transfer directly the connection from the Configuration Space to the Sizeand-Shape Space: some appropriate choices are needed.

As explained in details in $[18,20,47]$, the classical PT of form-vectors (or shape vectors) along geodesics is represented by the transport of horizontal vectors of
$\mathcal{C C}_{m}^{k}$ along horizontal geodesics (i.e. geodesics of $\mathcal{C C}_{m}^{k}$ whose tangent vectors are everywhere horizontal). In order to use here the same rationale, it is important to adapt the vertical and horizontal subspace splitting to the TPS metric. In fact the definition of the vertical subspace $\mathcal{V}_{X}$ does not depend on the metric, being determined directly by the tangent map $T \pi$ to the quotient map $\pi$. On the other hand the horizontal subspace $\mathcal{H}_{X}$, the orthogonal complement of $\mathcal{V}_{X}$, depends on the definition of direct sum related to the chosen metric.

Then, for any given point $X \in \mathcal{C C}_{m}^{k}$, the tangent space $T_{X}\left(\mathcal{C C}_{m}^{k}\right)$ splits, with respect to the quotient map $\pi$ from $\mathcal{E}^{k m}$ to $S \Sigma_{m}^{k}$, into a vertical and a horizontal subspace:

$$
\mathcal{T}_{X}\left(\mathcal{C C}_{k m}\right)=\mathcal{V}_{X} \oplus_{g} \mathcal{H}_{X}^{g}
$$

characterized as follows:

$$
\begin{gathered}
\mathcal{V}_{X}=\left\{V \in T_{X}\left(\mathcal{C C}_{m}^{k}\right): V=X W, W \in \operatorname{Skw}\left(\mathbb{R}_{m \times m}\right)\right\}, \\
\mathcal{H}_{X}^{g}=\left\{V \in T_{X}\left(\mathcal{C C}_{m}^{k}\right): \Gamma_{21 X} V \in \operatorname{Sym}\left(\mathbb{R}_{m \times m}\right)\right\} .
\end{gathered}
$$

In practice, a vertical vector at $X$ is an infinitesimal rotation of configuration $X$, while a TPS-horizontal vector is a vector whose uniform part is symmetric. Each TPS-horizontal vector represents a form-vector. A convenient way to define a PT transport of form vectors, compatible with the TPS metric and independent from the path is to select a representative section of the Sizeand-Shape Space and define the PT on it as follows:

- Assume a configuration P as Pole of the TPS Space.

- Select, for each form, an icon, defined as the configuration of the equivalence class of forms aligned with $\mathrm{P}$. The set of such icons is a section $\mathcal{S}_{P}$ of the quotient space $S \Sigma_{m}^{k}=\mathcal{C C}_{m}^{k} / S O(m)$, viewed as fibre bundle.

- Given two source configurations $X_{a}, X_{b} \in \mathcal{S}_{P}$ and a TPS-horizontal vector $V_{a}$ at $X_{a}$, we define the directly transported $V_{b}$ as the TPS-horizontal vector transported on $X_{b}$ with the TPS connection defined by $(7.21)$.

It is worth noting that, being a TPS-horizontal vector defined as a vector whose uniform part is symmetric and because TPS connection preserves the uniform component, the Direct Transport of a TPS-horizontal vector will still have a uniform part symmetric an then will be still a TPS-horizontal vector.

\section{Data Centering in the Size-and-Shape Space: Modified-Ordinary and Hierarchical Procrustes Analysis (MOPA \& HPA)}

In section 2 we introduced the OPA alignment and we observed that the difference between two aligned configuration is a standard horizontal vector. Now we need an 


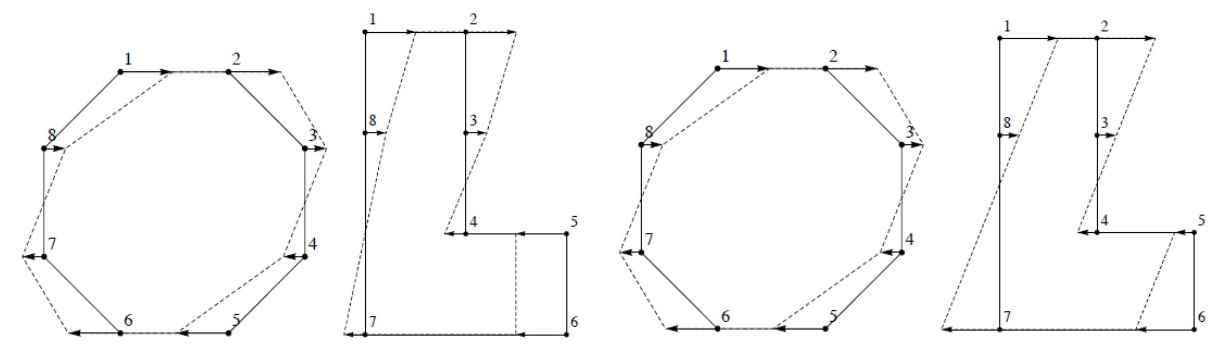

Fig. 1 The Levi Civita parallel transport (left) and a parallel transport that preserves the affine component (right) in the configuration space.
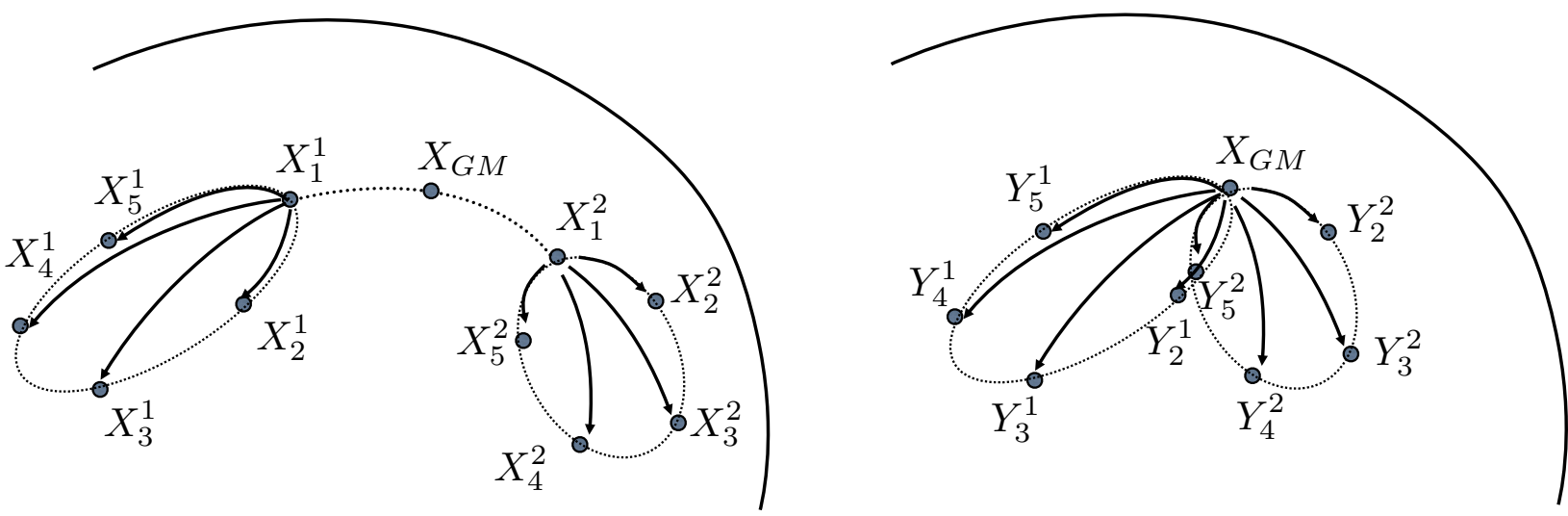

Fig. 2 Data Centering via Direct Transport. Left: Before shape data centering; Right: After the shape data centering.

alignment able to generate TPS-horizontal vectors. We name such types of alignment Modified OPA (MOPA). While OPA was based on the minimization of the Euclidean size-and-shape distance $d_{S}$, MOPA is based on the minimization of the TPS pseudo-distance defined as:

$d_{T P S}\left([X]_{S},[Y]_{S}\right)=\inf _{Q \in S O_{m}} \sqrt{g_{X}((Y Q-X),(Y Q-X))}$.

In particular the MOPA aligned configuration $\hat{X}_{b}$ is obtained by means of an optimal rotation $\hat{Q}$ minimizing $d_{T P S}$.

$\hat{Y}=Y \hat{Q}$

where $\hat{Q}=\operatorname{argmin} g_{X}((Y Q-X),(Y Q-X))$. According to this definition, $\hat{Q}$ turns out to be the rotational component of the polar decomposition of $(A-I)$, the TPS uniform component of the deformation vector $Y-$ $X$. Based on this definition, aligning a shape with another means filtering rotations out from the uniform part of the deformation; let us remark that rotations, as defined in a standard Procrustes alignment, are not deformation-based.

After the MOPA, the vector $\hat{Y}-X$ results as a TPS-horizontal vector of the tangent space on $X$. It is important to note that MOPA alignment makes sense only between near configurations, when the second can be considered as a small deformation of the first. On the other hand OPA alignment continues to be the main instrument to superimpose different bodies, characterized by very different shapes.

We now propose a Riemannian Data Centering to analyze sequences of configurations, based on the following algorithm (see Fig. 2). Let us consider $n$ different sequences $\left(X_{1}^{j}, X_{2}^{j}, X_{3}^{j}, \ldots\right)$, with $j=1, \ldots, n$; then:

1. Hierarchical Procrustes Analysis (HPA):

(a) Within each sequence, select a reference configuration $X_{c}^{j}$, which can be the first one, or the local mean;

(b) Perform a GPA with no scaling among the selected references $X_{c}^{j}$ to find the $X_{G M}$;

(c) Perform $n$ loops of OPA (or MOPA) with no scaling to unit CS, to align all the shapes of a sequence to its proper reference $X_{c}^{j}$.

2. Parallel transport:

(a) Build the TPS-horizontal (or horizontal) vectors $V_{i}^{j}=X_{i}^{j}-X_{c}^{j}$

(b) Transport the vectors toward $X_{G M}$ by using the TPS Direct Transport (or the Levi-Civita Parallel Transport along geodesics) in the Size-andShape Space; 
(c) add the transported vectors to the $X_{G M}$ to generate new sequences to be analyzed with standard methods: $Y_{i}^{j}=X_{G M}+V_{i}^{j}$.

The three steps of HPA may be considered as a preprocessing needed before transporting the configurations: Step 1a) is simply the choice of a reference configuration for each sequence; Step 1b) displaces each reference on the same section $\mathcal{S}_{G M}$ of the Size-andShape Space; Step 1c) aligns each configuration in a sequence with the reference, so that their differences become TPS-horizontal vectors (using MOPA) or horizontal vectors (using OPA).

In our case, with the TPS connection, after the MOPA based HPA, the formula to be used to transport TPS-horizontal vectors is (7.21).

For the standard LC connection, after the OPA based HPA, explicit PT formulas are available for two dimensional data; the formula to be used to transport the horizontal vector $V_{a}$ from $X_{a}$ toward $X_{b}$ is (see equation (3.2) in [47]):

$V_{b}=V_{a}-\frac{\sin \left(\theta_{a b}\right)\left(Z_{a}+Z_{b}\right) \Omega}{1+Z_{a} \cdot Z_{b}}, \quad \Omega=\left(\begin{array}{cc}0 & -1 \\ 1 & 0\end{array}\right)$

where $Z_{a}=X_{a} /\left\|X_{a}\right\|, Z_{b}=X_{b} /\left\|X_{b}\right\|, \theta_{a b}$ is the angle of the OPA alignment between $X_{a}$ and $X_{b}$.

At the end of the procedure, after the step $2 c$ ) we obtain a new set of sequences, to be analyzed with standard methods such as GPA+PCA.

\section{Preliminary examples: PT of ellipses}

As outlined in the introduction, in [32] it is proved that a non-degenerate scale-invariant metric does not exist for LDDMM. This imply that change in size and change in shape are coupled in PTs. The proof is illustrated by a simple example concerning the change in aspect ratio of a circle. It is shown that this simple deformation, once performed a PT upon LDDMM toward a smaller circle, the result is a much more circular shape: the ratio between the biggest and smallest axes decreases from 1.25 (before PT) to 1.18 (after PT). In order to correct this drawback the authors of [32] introduce two new models decomposing volume and shape variation. The behavior of the corrected models is then illustrated by means of a second simple example concerning the PT of the deformation (a scaling composed with a bump) of an ellipses toward a different closed curve. In the present section we replicate both the experiments by using our Direct Transport method (Figure 4). In both experiments both the area increment and aspect ratio are correctly transported. The same does not hold when using LDDMM according to [32].

\section{Case Study: Relating Deformations to PC scores}

Our purpose is to perform a reverse engineering experiment: at first, we define some parametrized deformation maps to be used to generate different sequences of shapes; then, by using size-and-shape analysis, we try to recover the values of the parameters that have been used to generate the sequences.

The experiment is made more challenging by using both sequences made with different reference configurations undergoing the same deformation, or sequences made of different configurations undergoing different deformations; our goal is to show the effects of intersubject variation and the capability of PCA in recovering deformation parameters.

As stated in the Introduction, one of the most used ordination technique is PCA performed on the covariance matrix of aligned coordinates. However, if there exist an inter-shape difference between the shapes deformed by different motions, the PCA will try to explain concomitantly both the intra- and inter- shape variations.

We discuss this issue by using an appropriate case study posed in the 2D Euclidean space; to generate a dataset of many different sequences, we consider a motion $\Phi_{c}$ described by two parameters $t \mapsto c(t)=$ $(\varepsilon(t), \gamma(t))$, and a set of five different reference configurations $X_{i}$, see Fig. 3, each sampled with 8 landmarks, assumed homologous; the two parameters $\varepsilon, \gamma$ represent two different modes of deformation, and may be used to define a uniform deformation, or a bending, see Fig. $3_{b}$. The uniform deformation $\Phi_{c}$ is represented through the matrix $F(t)$ as follows

$$
\begin{aligned}
& \left(\begin{array}{l}
x_{t} \\
y_{t}
\end{array}\right)=\left(\begin{array}{ll}
F_{11}(t) & F_{12}(t) \\
F_{21}(t) & F_{22}(t)
\end{array}\right)\left(\begin{array}{l}
x_{o} \\
y_{o}
\end{array}\right), \\
& \text { with } F(t)=\exp \left[\left(\begin{array}{cc}
\varepsilon(t) & 0 \\
0 & -\varepsilon(t)
\end{array}\right)+\left(\begin{array}{cc}
0 & \gamma(t) \\
\gamma(t) & 0
\end{array}\right)\right],
\end{aligned}
$$

and $\left(x_{o}, y_{o}\right)$ landmarks of $X_{i}$; here, $\varepsilon$ and $\gamma$ represent aspect ratio and shear, respectively. Let us note that $F$ maintains the area; note also that such a deformation is symmetric, and thus, it has a null rotational part. The non-uniform deformation $\Phi_{c}$ is represented by

$$
\left(\begin{array}{c}
x_{t} \\
y_{t}
\end{array}\right)=\frac{1+\gamma(t) \exp (\varepsilon(t)) x_{o}}{\gamma(t)}\left(\begin{array}{c}
\sin \left(\frac{\gamma(t) y_{o}}{\exp (\varepsilon(t))}\right) \\
\cos \left(\frac{\gamma(t) y_{o}}{\exp (\varepsilon(t))}\right)-1
\end{array}\right)
$$




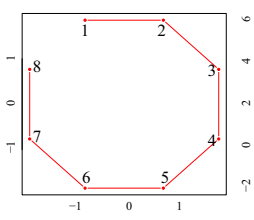

a) Parameters

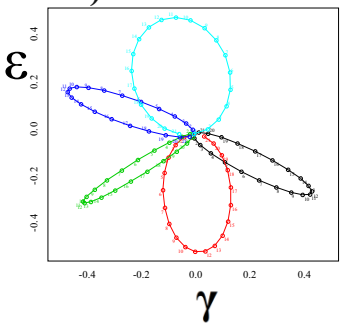

b) Aspect ratio

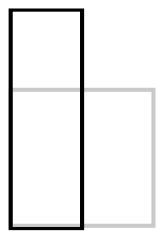

$\varepsilon$

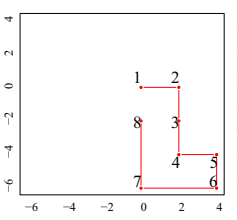

c) Affine: shear

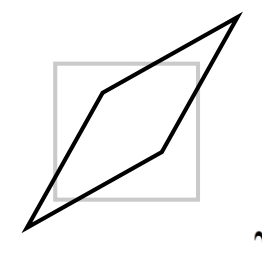

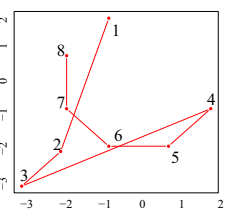

d) Non affine: bending

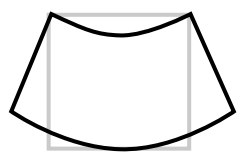

$\gamma$

Fig. 3 Top. Five reference Configurations. Bottom. Morphological meaning of deformation parameters. a) The $\varepsilon-\gamma$ space of parameters. b) effect of $\varepsilon$. c) effect of $\gamma$ in the affine case. d) effect of $\gamma$ in the non affine case.
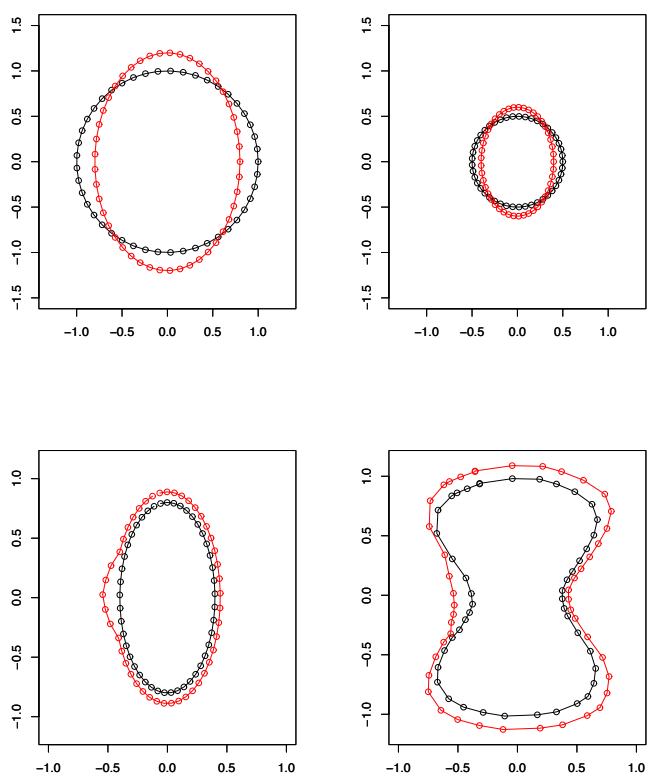

Fig. 4 Top left: A deformation of a circle defined by 45 landmarks with radius $=1$ into an ellipse with aspect ratio $=1.5$; area black circle: 3.13 ; area red ellipse 3.00. Top right: the deformation shown in top left panel applied to a circle with radius=0.5 and area $=0.78$. The resulting ellipse has an area $=0.75$ and aspect ration of 1.5 . The normalized area increment is conserved in both panel and it is approximately=-0.04. Bottom left: A local deformation of an ellipse; area black ellipse $=1.00$; area deformed ellipse $=1.28$. Bottom right: the same deformation of bottom left panel applied to a generic contour: area black (undeformed) contour $=2.11$; area red contour $=2.71$. In both case the normalized area increment $=0.28$. This experiment has been performed using DT and it is pretty comparable to the original one presented in [32].

here, $\varepsilon$ and $\gamma$ represent aspect ratio and bending curvature, respectively.

Both uniform and non uniform motions induce rotations, translations or scaling in a reference configuration; thus, the initial configurations undergoing the transformations are preliminary centered, scaled and optimally aligned via a common GPA. The sequences are generated by considering closed curves, called $c y$ - cles, in the space of parameters sampled at times $t_{i}=$ 1...21; we consider the following cases, see Fig.5:

- 1) One cycle $c(t)$ of uniform motions as in (11.24) applied to the five different reference configurations $X^{\ell}$ :

$\mathcal{T}_{F_{c}}\left(X^{\ell}\right)=\left(X_{t_{1}}^{\ell}, \ldots X_{t_{n}}^{\ell}\right)$,

with $X_{t_{i}}^{\ell}=F_{c}\left(t_{i}\right)\left(X^{\ell}\right)$; 
- 2) One cycle $c(t)$ of non-uniform motions as in eq. (11.25) applied to the five reference configurations $X^{\ell}$ :

$$
\mathcal{T}_{\Phi_{c}}\left(X^{\ell}\right)=\left(X_{t_{1}}^{\ell}, \ldots X_{t_{n}}^{\ell}\right)
$$

with $X_{t_{i}}^{\ell}=\Phi_{c}\left(t_{i}\right)\left(X^{\ell}\right)$;

- 3) Five cycles $c^{j}(t)$ of uniform motions as in 11.24 applied to the five reference configurations $X^{\ell}$, posing $\ell=j$, that is, each configuration $X^{j}$ is deformed with cycle $c^{j}(t)$ :

$\mathcal{T}_{F_{c j}}\left(X^{j}\right)=\left(X_{t_{1}}^{j}, \ldots X_{t_{n}}^{j}\right)$

with $X_{t_{i}}^{j}=F_{c^{j}}\left(t_{i}\right)\left(X^{j}\right)$;

- 4) Five cycles $c^{j}(t)$ of non-uniform motions as in (11.25) applied to the five reference configurations $X^{\ell}$, with $\ell=j$, that is, each configuration $X^{j}$ is deformed with cycle $c^{j}(t)$ :

$$
\begin{aligned}
\mathcal{T}_{\Phi_{c^{j}}}\left(X^{j}\right) & =\left(X_{t_{1}}^{j}, \ldots X_{t_{n}}^{j}\right), \\
\text { with } X_{t_{i}}^{j} & =\Phi_{c^{j}}\left(t_{i}\right)\left(X^{j}\right) .
\end{aligned}
$$

In order to simulate more realistic datasets, a random uniform rotation is applied to each configuration of the generated data. Let us note that cases 1 and 2 are meant to assess if our procedure is able to recognize that the five different sequences in $\mathcal{C C}_{m}^{k}$ have been generated by a same cycle of parameters $c(t)$, despite the differences among the reference configurations. On the other hand all the cases are meant to assess if our procedure is able to

- recognize that all the sequences are generated by the combination of only two deformation modes (parameters)

- reconstruct the whole pattern of the five cycles in the plane of the two parameters (form of the cycles and reciprocal orientation)

The analysis of each case is performed by comparing three different methods:

1. Classic: no preliminary data centering

2. LC Data Centering: preliminary data centering based on the Levi Civita parallel transport in Size-andShape Space

3. DT Data Centering: preliminary data centering based on our TPS Direct Transport of in the Size-andShape Space

In each case a GPA followed by a PCA is then performed.

We quantify the ability in recovering original cycles of PCAs by adopting the so called trajectory analysis (see $[6,35])$. In practice we consider each cycle in the $(\varepsilon, \gamma)$ plane as a shape itself, where pairs $\left(\varepsilon\left(t_{i}\right), \gamma\left(t_{i}\right)\right)$ identify the landmarks. We thus have two main shapes each with 105 landmarks: an elliptical shape constituted by 5 petals perfectly superimposed and a "flower" shape constituted by 5 radially oriented petals. This allows us to comparing these shapes with those identified in the space of first two PCs from the PCAs of the analyses that we evaluated in this study. Thus, the ability in recovering the parameter space of the methods presented here is evaluated in terms of non-uniform component of the partial Procrustes distances between original parameters shapes (the elliptical shape constituted by the 5 identical cycles or the "flower" shape) and the shapes identified by the first two PCs in the PC space of the corresponding analyses based on classic approach, DT and LC parallel transports. This choice is due to the fact that a simple linear re-parameterization of the assigned deformations could change the aspect ratio of the plane $\varepsilon, \gamma$, thus the ability in recovering them is quantified only upon non uniform deformation. This partial Procrustes distance is normalized on the maximum distance allowed that is $\sqrt{2}$.

The comparison of results obtained by means of the three methods is plotted in Fig. 6, where the first two $\mathrm{PC}$ scores are shown. In the same figure the deformation modes corresponding to each PC are shown. Table 2 and Table 3 report, respectively, the global variance explained by the first two PC scores and the Procrustes Distance between original parameters shapes and the shapes of cycles identified by the first two PC scores. Fig. 7 illustrates the Procrustes superimposition of non uniform components of deformations between original cycles and cycles recovered after GPA on the shape space+PCA performed on data transported according to DT (top) or LC (bottom). Shape distances (normalized by $\sqrt{2}$ ) between red and black shapes are shown in Table 3 .

Table 2 Variance explained by firsts two PC scores

\begin{tabular}{lllll} 
& case 1 & case 2 & case 3 & case 4 \\
\hline Classic & $90 \%$ & $67 \%$ & $87 \%$ & $88 \%$ \\
LC & $76 \%$ & $80 \%$ & $82 \%$ & $84 \%$ \\
DT & $100 \%$ & $96 \%$ & $100 \%$ & $87 \%$
\end{tabular}

Table 3 Partial Procrustes Distance between non uniform components of the original and reconstructed parameters cycles pattern ( $\%$ of the maximum $d_{P}=\sqrt{2}$ )

\begin{tabular}{lllll} 
& case 1 & case 2 & case 3 & case 4 \\
\hline Classic & $70 \%$ & $69 \%$ & $61 \%$ & $67 \%$ \\
LC & $54 \%$ & $42 \%$ & $19 \%$ & $26 \%$ \\
DT & $1.7 \%$ & $18 \%$ & $11 \%$ & $20 \%$
\end{tabular}




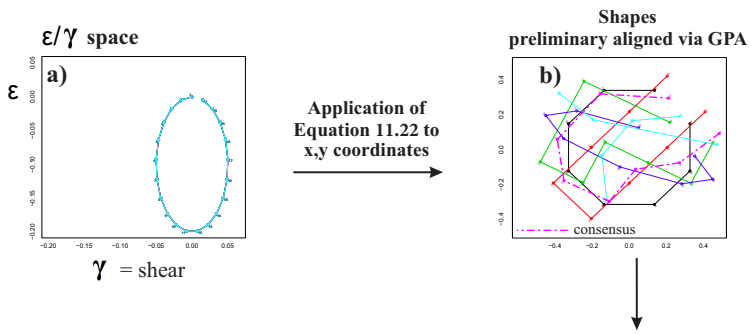

CASE 1

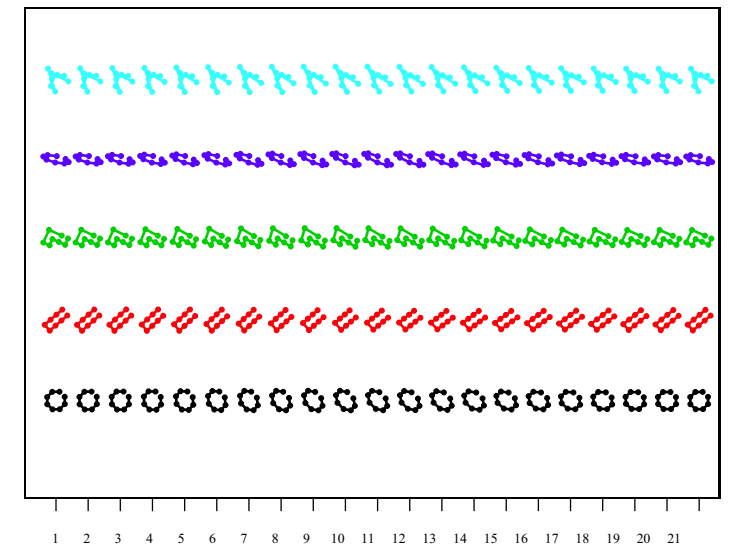

Deformational step

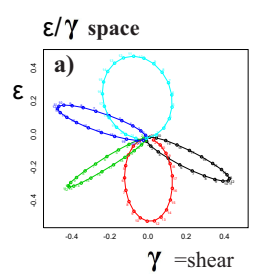
$\stackrel{\begin{array}{c}\text { Application of } \\ \text { Equation } 11.22 \text { to } \\ \mathbf{x}, \mathbf{y} \text { coordinates }\end{array}}{\longrightarrow}$

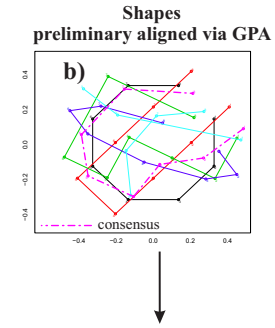

CASE 3

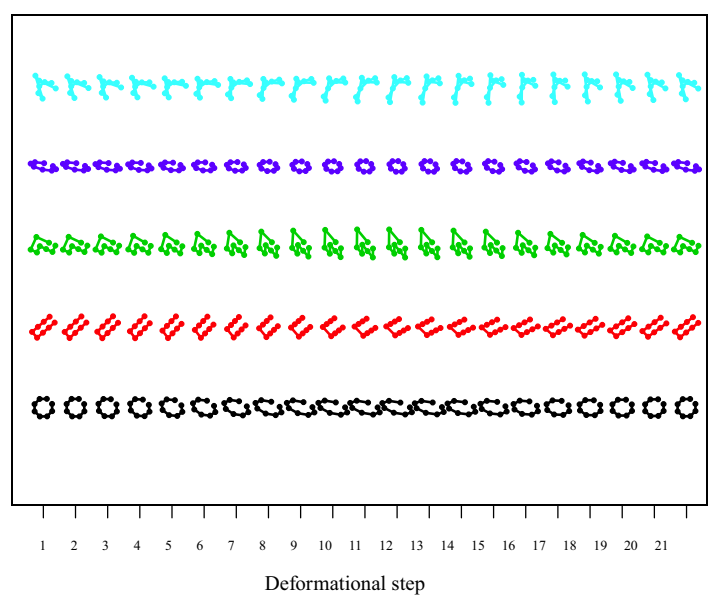

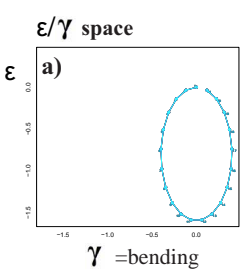
Application of
Equation 11.23 to x,y coordinates

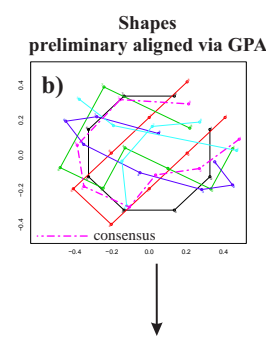

CASE2

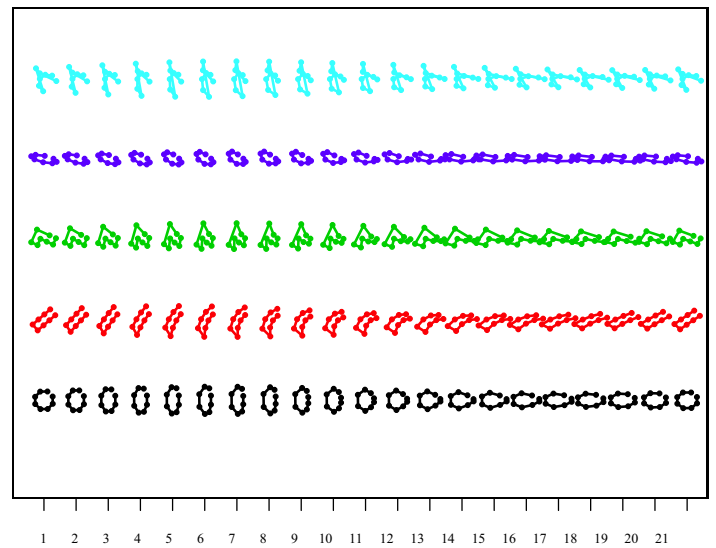

Deformational step

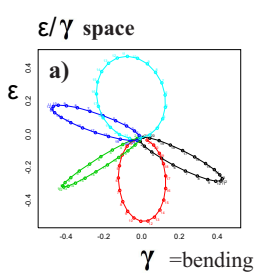

Application of Equation 11.23 to

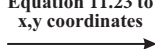

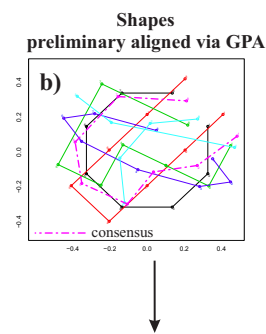

CASE 4

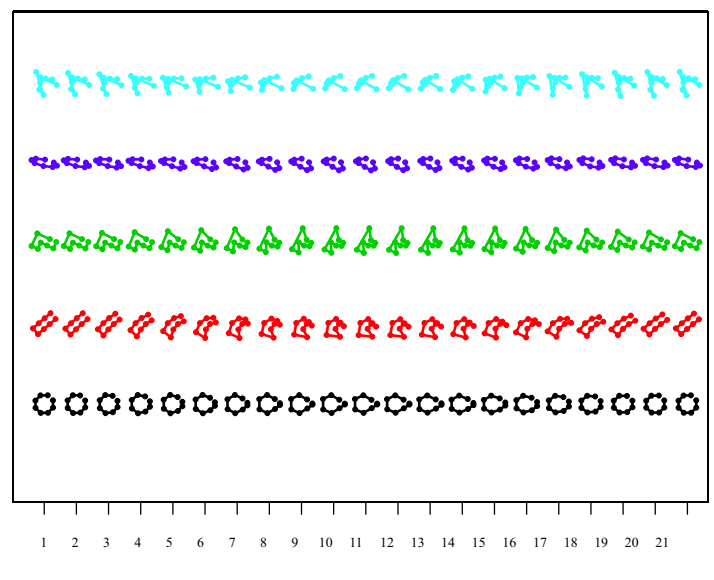

Deformational step

Fig. 5 Dataset generation under different conditions. Case 1 and case 3 are affine cases; case 2 and case 4 are non affine cases.

In case 1 the DT method recovers virtually perfectly the parameters cycles:
- the five cycles in the PCs space plane are superimposed

- the $100 \%$ of the variance is explained by two PCs 
- both the modes corresponding to that PCs are affine

- the normalized Procrustes distance between the cycles pattern is only $2 \%$

We note that both for Classic and LC methods the five cycles are not superimposed, the performance measures (variance explained and procrustes distance) are worst than in the DT method. Moreover, in both cases the deformation modes represented by the first two PC are not uniform. In the first case this happens because the PCs have to explain first of all the difference between the initial configurations. In the second case is crucial the fact that LC connection does not distinguish between uniform and non uniform component.

In case 2 the DT method is not perfect as in case 1 but it results in being the best among the three methods, in fact

- the five cycles in the PCs space are quasi superimposed

- the $96 \%$ of the variance is explained by the first two $\mathrm{PCs}$

- the modes corresponding to $\mathrm{PC}_{1}$ is uniform while that corresponding to the $\mathrm{PC}_{2}$ is nonuniform (bending)

- the normalized Procrustes distance of the shape of the cycles pattern from the "flower" shape of original parameters is higher $(18 \%)$ than for case 1 but much lower that in the other two methods

In case 3 the DT method performs again very well:

- the $100 \%$ of the variance is explained by firsts two PCs

- both the modes corresponding to that PCs are affine

- the normalized Procrustes distance of the shape of the cycles pattern from the flower" shape of original parameters is small: $10 \%$

In case 4 the DT method results in being the best of the three methods even if LC method appears quite acceptable. On the the other hand the classic approach returns, as in the other cases, cycles that account mostly for inter-group differences without any recovery of deformation parameters.

\section{Examples with real data: Left ventricle analysis}

We now consider data coming from 3D echocardiography on 48 real human left ventricles (LV) moving in time. These data come from the same research project partially published in $[35,47]$. In those papers it was shown that the trajectory analysis is able, only after a proper data centering (there based on LC connection), to distinguish between healthy and pathological subjects. Here, taking for granted that result, we will show as the DT can give a more accurate transport of the deformations compared to LC. We collected shape data by means of 3D Speckle Tracking Echocardiography (PST25SX Artida, Toshiba Medical Systems Corp., Tokyo, Japan). The result of our 3DSTE system is a time-sequence of configurations, each constituted by 1297 landmarks, assumed to be homologous. These 48 motion trajectories belong to healthy individuals and were acquired at the same electromechanically homologous times used in $[35,47]$. However, in order to better interpolate motions, we used a finer homologous time sampling. This results in individual motion trajectories each composed by 16 homologous times. The homology of landmarks is ensured by the fact that six landmarks are manually digitized by the operator (the same for all subjects) on the apex, the base, the mitral annulus and the interventricular septum. Starting from these landmarks the rest of the cloud is generated via speckle tracking as interlandmarks that are topologically homologous across different individuals.

It is worth noting that formula (9.23) holds in $2 \mathrm{D}$; thus, in order to compare our procedure with the LC data centering, we generate a $2 \mathrm{D}$ dataset by projecting a coronal slice of the epicardial and endocardial 3D landmarks on the plane (that passing trough interventricular septum) transversal to the LV base identified by the diameter of mitral annulus and ventricular apex.

For the obtained dataset we perform a shape analysis using the same three methods of the previous simulated examples.

It is worth noting that in this example we are not performing reverse engineering, so we cannot evaluate the performance of the used methods comparing the obtained results with some a priori known solution. Nevertheless, we can compare the performance of the parallel transport methods by checking the conservation of some important quantities related to the deformation when one passes from the original to the centered data. In particular we check:

- bending energy estimated from the individual enddiastolic states (relaxed states) to each of the individualspecific deformed state.

- Procrustes distance between the individual end-diastolic states (relaxed state) and each of the individualspecific deformed states.

- As a last check we performed separate GPAs in the shape space followed by PCA. We then stacked in single vectors all PC1 or PC2 or PC3 scores coming from these PCAs. These vectors represents the 

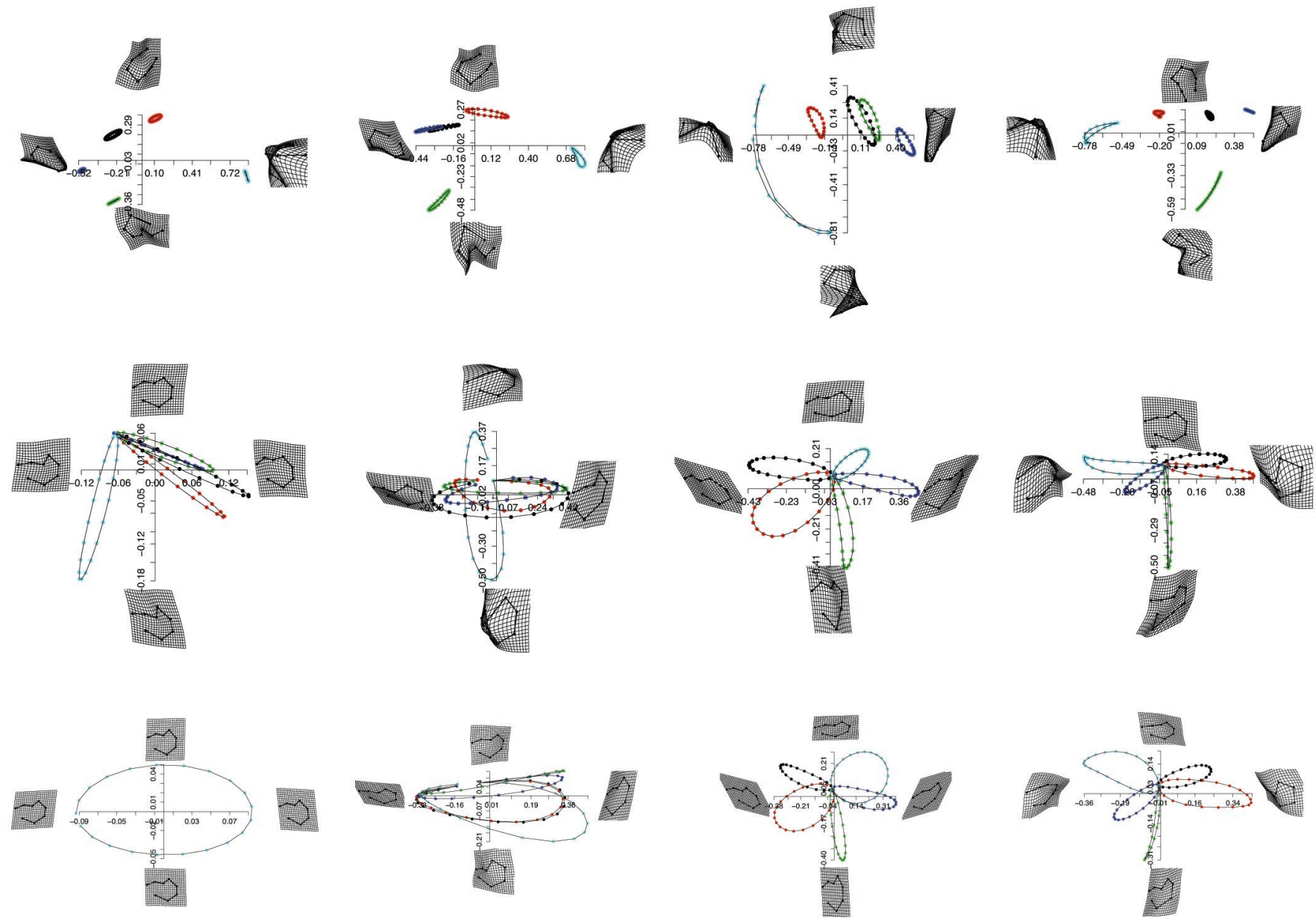

Fig. 6 The comparison of results of classic GPA+PCA, Levi Civita Riemannian Parallel Transport in size and shape space, and Direct Transport. The first two PC scores are shown. From top to bottom the three methods and from the left to the right the four cases are reported.

"true" individual deformation cycles not affected by the presence of other individuals. We plotted these vectors against $\mathrm{PC} 1$ or $\mathrm{PC} 2$ or PC3 scores, stacked in the same way, coming from separate GPAs+PCAs performed after LC or DT parallel transports. For any individual motion trajectory (each composed by
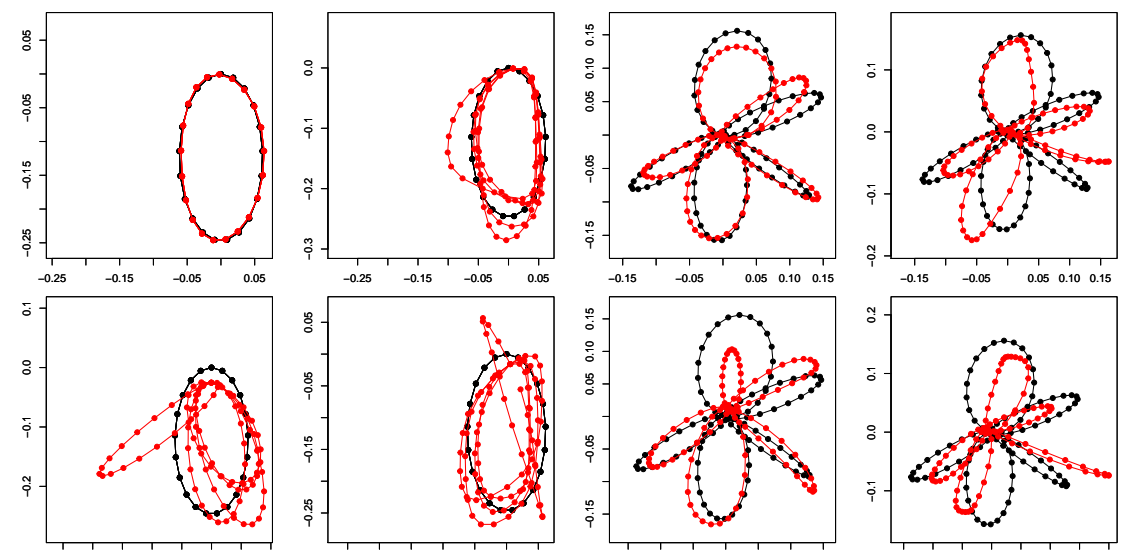

Fig. 7 Procrustes superimposition of non uniform components of deformations between original cycles and cycles recovered after GPA on the shape space+PCA performed on data transported according to DT (top) or LC (bottom). 
16 shapes) we computed the absolute deviation from isometry $(=1)$ of regression coefficient between PCs of pure data and transported data (either DT or LC). We then performed ANOVAs using DT/LC as factor variable in order to assess significance.

Fig. 8 reports the results of the PCA performed on real data using the three different methods, together with the deformation modes. While the classic method still shows evidence of the ambiguous meaning of PCs that explain concomitantly intra- and inter- individual variation, the LC and the DT yield qualitatively similar results. While for the classic method there is no hope in observing the sole deformation cleaned up by inter-individual variability, the deformations illustrated by LC and DT are coherent. The PC1 represents the global contraction and the myocardial thickening, while PC2 is associated with different extents of deformation affecting the mitral annulus and ventricular apex. We note that the initial shapes we used in the simulated datasets were intentionally challenging as they are hugely different. In fact, the maximum geodesic Procrustes distance between pairs of shapes in our simulated datasets is about 1.2 (the maximum allowed is about $1.57 \simeq \pi / 2$ ). Our real data span 0.25 of geodesic Procrustes distance thus making the use of LC connection still acceptable. Despite this qualitative similarity, some significant differences emerge looking at Fig.9, where the bending energy, the Procrustes distance (both calculated for each shape of each individual from its proper end-diastolic state) and the first three PC scores coming from separate GPAs+PCAs before the data centering are plotted versus the same quantities after the data centering via DT or LC. It is evident that our DT method conserve both the bending energy and the Procrustes distance better than LC. An alternative representation (per-individual absolute deviations from isometry) of the same results is given in Fig. 10. There are illustrated the absolute deviations from isometry $(=1)$ of regression' beta coefficients for data shown in Fig.9. Regressions were calculated using the model DT or LC result $\sim$ Original result for each of the individual cycles. ANOVAs results are always significant (=asterisks). DT method performs always better than LC. It is worth noticing that for bending energy the absolute deviation from isometry for regression' beta coefficients is zero as a consequence of the definition of DT (eq.7). The averaged values of absolute deviations from isometry for DT are 0.027, 0.025, 0.032 for PC1, PC2 and PC3 respectively, while they are 0.12, 0.094, 0.093 for LC parallel transport. In order to assess significance we performed ANOVAs on absolute deviations from isometry using DT/LC as factor variable. We found differences always significant ( $p$-value for PC1: 1.972e-10; p-value for PC2: 1.857e-07; p-value for PC3: 2.491e-06). In addition Levene's test revealed that DT always shows a significant smaller variance respect to LC (PC1: 5.6e-4 vs 7.9e-3 [p-value: 6.94e-7]; PC2: 3.6e4 vs $6.8 \mathrm{e}-3$ [p-value: $2.98 \mathrm{e}-6$ ] PC $3: 9.5 \mathrm{e}-4$ vs. $6.2 \mathrm{e}-3$ [pvalue: $8.84 \mathrm{e}-5]$ ). From these results it is evident that DT preserves original deformations better than LC even if the latter might be acceptable.

\section{Conclusions}

In this paper we considered the problem of comparing trajectories of forms in presence of inter-group differences. We proposed to solve this problem by performing a data centering in the Riemannian space, by means of a connection characterized by a parallel transport that preserves the original components of the deformations. In particular, we pointed out that, in order to build such type of connection is not sufficient to provide a metric and the related Levi-Civita connection, but it is necessary to introduce a connection with torsion, named TPS connection leading to parallel transport named Direct Transport. By means of a set of simulations we performed a reverse engineering experiment and we showed that in the uniform motion (case 1 and 3) the original deformation cycles are perfectly recovered by the Direct Transport procedure. In case of non uniform motions (case 2 and 4) the DT method is not perfect as in uniform cases but it performs very well and results in being the best among the three methods.

We want to stress that in the non affine case a perfect example of reverse engineering cannot be built. This depends on the fact that applying the same diffeomorphism to different bodies does not mean applying the same deformation. To be more precise a diffeomorphism, e.g. (11.25), transforms the ambient space and the bodies follow it. But in this way each landmark moves accordingly to its position in the space. No covariation and reciprocal position among landmarks is considered in this way. The deformation can be characterized by a global component (affine) and a series of local components. In order to transport the global component one can transform the whole space, by ignoring the role of the landmarks, but the local component is related to the single landmark neighborhood. Homology cannot be neglected in the non-affine case (see [44] for the non landmark based case). The only way to apply the same non-uniform deformation to different bodies would be to assign a diffeomorphism to a first body and to transport the obtained deformation vector toward the other four bodies by means of the TPS connection. But in this way the reverse engineering falls in a circular reasoning: recovering via Direct 
Transport a dataset generated by Direct Transport. On the other hand the homology is naturally embedded in the real cases, then we consider as the most important non-uniform example in the present paper the real one: human left ventricles. Despite the small Procrustes distances encompassed by the entire left ventricular data, the performance indicators presented show an evident superiority of the DT method with respect to the others.

\section{Acknowledgments}

We are grateful to Lena R. Zastrow and Antonio DiCarlo for hints and advices; their helpful discussions stimulated us to go far beyond our initial intuitions. The authors wish also to express their gratitude to Willem Gorissen, Clinical Market Manager Cardiac Ultrasound at Toshiba Medical Systems Europe, Zoetermeer, The Netherland, for his continuous support and help. We thank Antonio Profico for his unvaluable sup- port in building deformetrics $\mathrm{R}$ package. We also thank three anonymous reviewers for their help in improving the manuscript. L.T., V.V. and S.G acknowledge the National Group of Mathematical Physics (GNFMINdAM), Italy, for support.

\section{References}

1. Bookstein, F. L. 1989. Principal Warps: Thin-Plate Splines and the Decomposition of Deformations. Journal IEEE Transactions on Pattern Analysis and Machine Intelligence archive, Vol.11 Issue 6: 567-585.

2. Bookstein, F. L. 1991. Morphometric tools for landmark data: Geometry and biology. Cambridge: Cambridge University Press.

3. Bookstein, F. L. 1997. Two shape metrics for biomedical outline data: Bending energy, Procrustes distance, and the biometrical modeling of shape phenomena, Proceedings of 1997 International Conference on Shape Modeling and Applications.

4. Pokrass J., M. Bronstein A., Bronstein M. M., Partial shape matching without point-wise correspondence, $\mathrm{Nu}-$
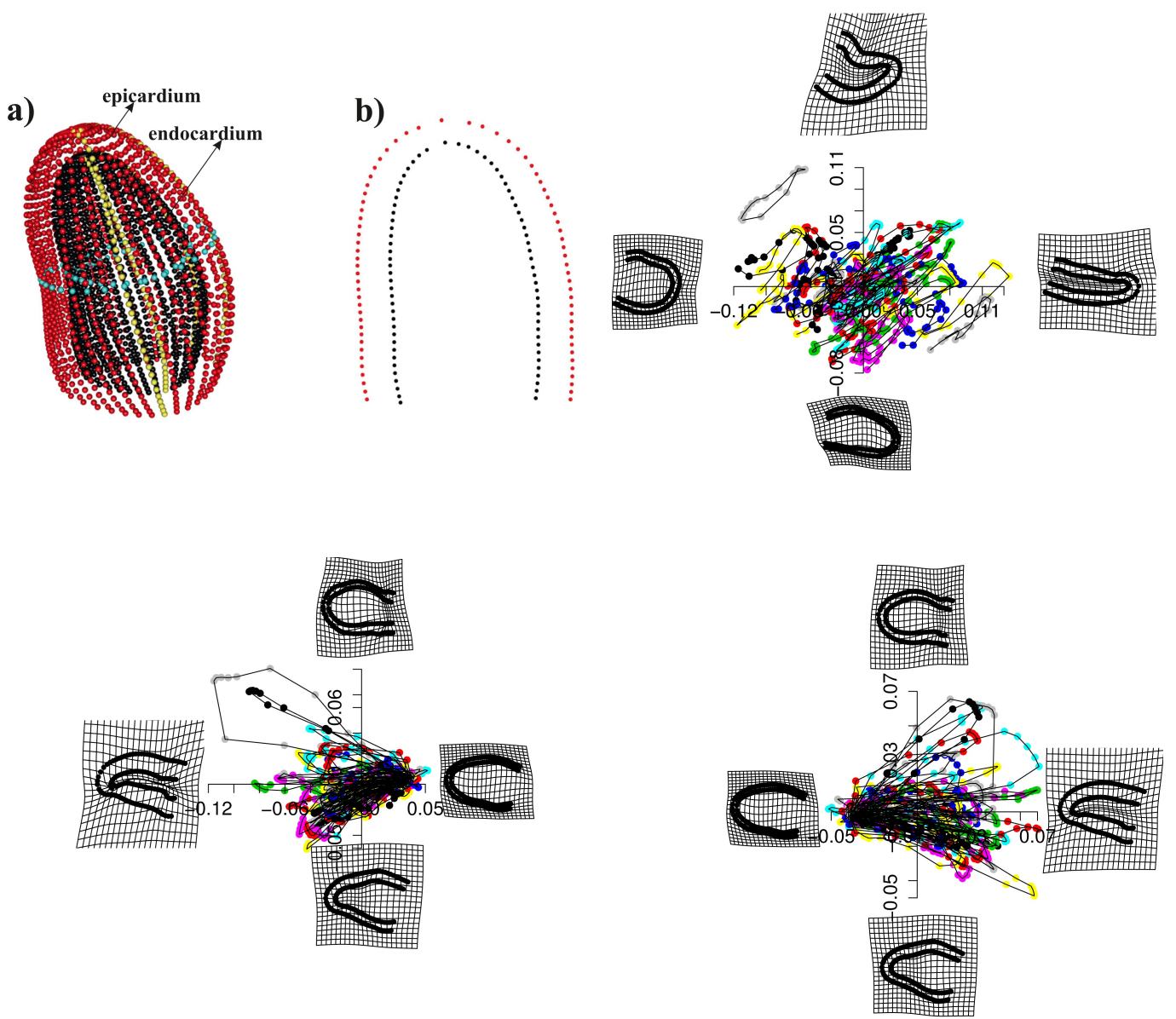

Fig. 8 The real example. Top left: the projection in 2D of the actual three-dimensional ventricular shape. Top right: classic GPA in the shape space+PCA. Bottom left: LC paralell transport in the size and shape space+GPA in the shape space+PCA. Bottom right: DT in the size and shape space+GPA in the shape space+PCA. Colors indicate the five deformations cycles belonging to simulated shapes. Colors indicate the individual cycles each composed by 16 shapes. 

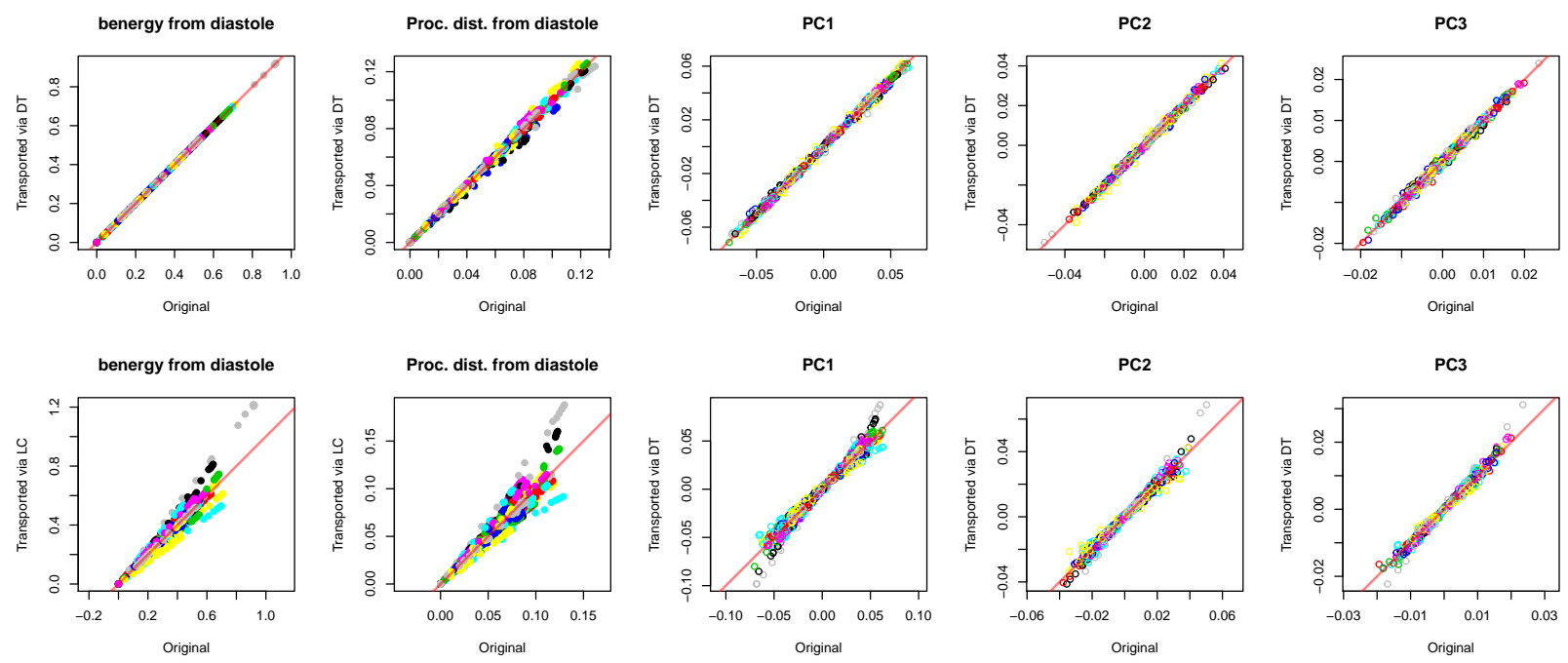

Fig. 9 Performance evaluation of the real example. Top row: DT; bottom row: LC. From left to right: i) scatterplot between bending energies estimated from the individual-specific end-diastolic states (relaxed states) and each of the individual-specific deformed state calculated on pure original data (x-axis) and on data transported via DT (top) or LC (bottom). ii) The same as above with Procrustes distances. iii) The same as above for the fisrt three PCs coming from separate GPAs+PCAs on original data (x-axis) and those coming from separate GPAs+PCAs performed on transported data via DT (top) or LC (bottom). Colors indicate the individual cycles each composed by 16 shapes.

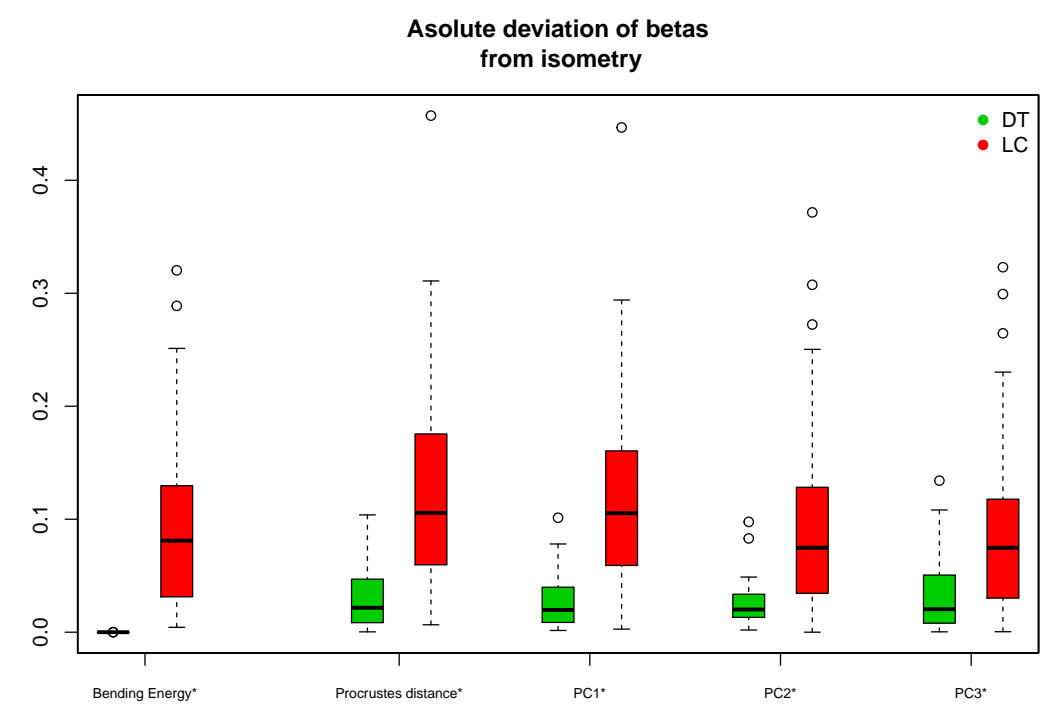

Fig. 10 Absolute deviations from isometry $(=1)$ of regression' beta coefficients for data shown in Fig.9. Regressions were calculated using the model DT or LC result Original result for each of the individual cycles. ANOVAs are always significant (=asterisks). DT method perfoms always better than LC. It is worth noticing that for bending energy the absolute deviation from isometry for regression' beta coefficients is zero as a consequence of the definition of DT (eq.7).

merical Mathematics: Theory, Methods and Applications (NM-TMA), Vol. 6/1, pp. 223, 2013

5. B.Charlier, N.Charon, A.Trouvé. 2015. The fshape framework for the variability analysis of functional shapes, Foundations of Computational Mathematics 1-71

6. Collyer M.L., Adams D.C. 2013. Phenotypic trajectory analysis: comparison of shape change patterns in evolution and ecology. Hystrix, 24, 75-82.
7. Cootes T. F., Twining C. J., Babalola K. O., Taylor C. J. 2008. Diffeomorphic statistical shape models. Image Vision Comput. 26(3):326-332

8. Crampin M., Pirani F.A.E. 1986 Applicable Differential Geometry, Cambridge University Press.

9. Dryden, I.L., Mardia, K.V. 1998 Statistical Shape Analysis, Wiley.

10. Nicolas Duchateau, Mathieu De Craene, Xavier Pennec, Beatriz Merino, Marta Sitges, Bart Bijnens. Which Re- 
orientation Framework for the Atlas-Based Comparison of Motion from Cardiac Image Sequences? Spatio-temporal Image Analysis for Longitudinal and Time-Series Image Data. Volume 7570 of the series Lecture Notes in Computer Science pp 25-37. 2012.

11. Erikson, A. P., and Astrom, K. 2012. On the bijectivity of thin-plate splines. Anal. Sc. Eng. 6: 93141.

12. J.B. Fiot, L. Risser, L.D. Cohen, J. Fripp, F.X. Vialard. 2012. Local vs global descriptors of hippocampus shape evolution for Alzheimer's longitudinal population analysis 2nd International MICCAI Workshop on Spatiotemporal Image Analysis for Longitudinal and Time-series Image Data (STIA '12), Nice, France (2012), pp. 1324

13. J. Glaunès. Transport par diffomorphismes de points, de mesures et de courants pour la comparaison de formes et lanatomie numrique. PhD thesis, Universit Paris 13, 2005.

14. Huckemann S., Hotz T., Munk A. 2010. Intrinsic MANOVA for Riemannian Manifolds with an Application to Kendall's Spaces of Planar Shapes. IEEE Trans. Patt. Anal. Mach. Intell., 32 (4): 593-603.

15. Kendall, D.G. 1977. The diffusion of shape. Advances in Applied Probability 9 (3): 428430.

16. Kendall, D. G., Barden D., Carne T. K., Le H. 1999. Shape and Shape Theory, Wiley.

17. Klingenberg W. 1982. Riemannian Geometry, Walter de Gruyter, Berlin, New York.

18. Kume A., Dryden, I.L., Le H. 2007. Shape space smoothing splines for planar landmark data. Biometrika, 94: 513528.

19. Le H., Kume A. 2000. Detection of shape changes in biological features. Journal of Microscopy, 200 (2): 140147.

20. Le H. 2003. Unrolling shape curves, J. London Math. Soc. (2) 68: 511526

21. Doug M. Boyer, Yaron Lipman, Elizabeth St. Clair, Jesus Puente, Biren A. Patel, Thomas Funkhouser, Jukka Jernvall, and Ingrid Daubechies,Algorithms to Automatically Quantify the Geometric Similarity of Anatomical Surfaces, PNAS, vol. 108 no. 45, November 8, 2011

22. Lorenzi, M. and Pennec, X. Efficient Parallel Transport of Deformations in Time Series of Images: from Schild's to Pole Ladder. Journal of Mathematical Imaging and Vision, Springer Verlag, 2013, 50 (1-2), pp.5-17.

23. Lorenzi, M. and Pennec, X. Geodesics, parallel transport and one-parameter subgroups for diffeomorphic image registration. International Journal of Computer Vision (2013) 105: 111-127.

24. Lorenzi, M., Ayache, N., Frisoni, G.B., and Pennec X. Mapping the effects of $\mathrm{A} \beta_{1-42}$ levels on the longitudinal changes in healthy aging: hierarchical modeling based on stationary velocity fields. In Proceedings of Medical Image Computing and Computer Assisted Intervention (MICCAI), volume 6892 of LNCS, pages 663-670, 2011. Springer

25. Marsland S., Twining C., Principal Autoparallel Analysis: Data Analysis in Weitzenbck Space, 2015. https://arxiv.org/abs/1511.03355

26. Charles-Michel Marle, The works of Charles Ehresmann on connections: from Cartan connections to connections on fibre bundles, BANACH CENTER PUBLICATIONS, VOLUME 76, POLISH ACADEMY OF SCIENCES, WARSZAWA 2007. https://arxiv.org/abs/1401.8272

27. Miller M.I., Younes L. 2001. Group actions, homeomorphisms, and matching: A general framework. International Journal of Computer Vision, 41 (1-2): 61-84.

28. Miller M. I., Qiu A., 2009. The emerging discipline of Computational Functional Anatomy. Neuroimage 45 516539
29. Miller, M. I., Mori, S., Qiu, A., Zhang, J., and Ceritoglu, C. 2013. Advanced cost functions for image registration for automated image analysis: Multi- channel, hypertemplate, and atlas with built-in variability. Patent n. US 8600131 B2.

30. M. Miller, L. Younes and A. Trouvé. Diffeomorphometry and geodesic positioning systems for human anatomy. Technology 02,(36), 2014.

31. M. Miller, L. Younes and A. Trouve. Hamiltonian Systems in Computational Anatomy: 100 Years since D'Arcy Thompson. Annual Review of Biomedical Engineering 17 2015

32. Niethammer M, Vialard F.X. 2013. Riemannian metrics for statistics on shapes: parallel transport and scale invariance. Proceedings of the 4th MICCAI workshop on Mathematical Foundations of Computational Anatomy (MFCA). 1-13

33. Xavier Pennec, Marco Lorenzi. Which parallel transport for the statistical analysis of longitudinal deformations? Colloque GRETSI '11, 2011, Bordeaux, France. 2011.

34. Peter A. M., Rangarajan A. 2009. Information Geometry for Landmark Shape Analysis: Unifying Shape Representation and Deformation. IEEE Trans Pattern Anal Mach Intell. 31(2): 337350.

35. Piras P., Evangelista A., Gabriele S., Nardinocchi P., Teresi L., Torromeo C., Schiariti M., Varano V., Puddu P. E. 2014. 4D-Analysis of Left Ventricular Heart Cycle Using Procrustes Motion Analysis. PLoS ONE 9(1): e86896.

36. Qiu, Albert, Younes, Miller. Time sequence diffeomorphic metric mapping and parallel transport track timedependent shape changes. Neuroimage. 2009 Mar;45(1 Suppl):S51-60. doi: 10.1016/j.neuroimage.2008.10.039. Epub 2008 Nov 7.

37. Rohlf F.J. and Bookstein, F. L., Computing the Uniform Component of Shape Variation. Systematic Biology 52(1):66-69, 2003.

38. J.A. Schouten, Ricci calculus, Springer-Verlag (1954).

39. Spivak M. 1999. A Comprehensive Introduction to Differential Geometry, Vol.2, Third Edition, PUBLISH or PERISH, INC. 06 Houston, Texas.

40. Srivastava A., Klassen E., Joshi S.H., Jermyn I.H., 2011. Shape Analysis of Elastic Curves in Euclidean Spaces, Pattern Analysis and Machine Intelligence, IEEE Transactions on, 33(7), pp.1415-1428.

41. Sundaramoorthi, G., Mennucci, A., Soatto, S. and Yezzi, A., 2011. A new geometric metric in the space of curves, and applications to tracking deforming objects by prediction and filtering. SIAM Journal on Imaging Sciences, 4(1), pp.109-145.

42. Tang, X., Holland, D., Dale, A. M., Younes, L., and Miller, M. I. 2015. The diffeomorphometry of regional shape change rates in mild cognitive impairment and Alzheimers disease. Hum. Brain Mapp. 36: 20932117.

43. A. Trouvé and L. Younes. 2005. Metamorphoses Through Lie Group Action, Foundations of Computational Mathematics $173-198$

44. Twining, C., Marsland, S., Taylor, C.: Metrics, connections, and correspondence: the setting for groupwise shape analysis. In: Energy Minimization Methods in Computer Vision and Pattern Recognition. pp. 399-412. Springer (2011)

45. Trouvé A. 1995. An approach to pattern recognition through infinite dimensional group action. Technical Report, Ecole Nationale Superieure, Université Paris 6, Departement de Mathématiques et Informatique, Laboratoire d'Analyse Numerique. 
46. Vaillant, M., Glaunès, J., 2005. Surface matching via currents. In G. Christensen and M. Sonka (eds.). IPMI, Ser. Lecture Notes in Computer Science. Springer-Verlag, Berlin Hidelberg, pp. 381-392.

47. Varano V., Gabriele S., Teresi L., Dryden I., Puddu P. E., Torromeo C., and Piras P. (2015) Comparing Shape Trajectories of Biological Soft Tissues In The Size-AndShape Space. BIOMAT 2014: pp. 351-365.

48. Xie Q., Kurtek S., Le H., Srivastava A. 2013. Parallel Transport of Deformations in Shape Space of Elastic Surface, IEEE Int. Conf. on Computer Vision (ICCV), Sydney, Australia.

49. Yezzi, A.J. and Soatto, S., 2003. Deformotion: Deforming motion, shape average and the joint registration and approximation of structures in images. International Journal of Computer Vision, 53(2), pp.153-167

50. Younes, L.: Jacobi Fields in groups of diffeomorphisms and applications. Q. Appl. Math pp. 113-134 (2007)

51. Younes, L., Qiu, A., Winslow, R., Miller, M.: Transport of relational structures in groups of diffeomorphisms. J. Math Imaging Vis 32(1), 41-56 (2008)

\section{Appendix}

In the following we derive the right value of $\nu$ coefficient used for quantifying the absolute value of the bending energy (eq. 5.13, 5.14).

We start from the equation (3.2) from KENT, J. T. \& MARDIA, K. V. (1994). The link between kriging and thin plate splines. In Probability, Statistics and Optimization, Ed. F. P. Kelly, pp. 324-39. New York: Wiley.

$$
\begin{aligned}
\sigma_{\alpha}(h) & =c_{\alpha, d}|h|^{2 \alpha} & & \alpha>0, \alpha \text { not an integer } \\
\sigma_{k}(h) & =b_{k, d}|h|^{2 k} \log |h| & \alpha & =k, k>0 \text { an integer }
\end{aligned}
$$

where

$c_{\alpha, d}=2^{-2 \alpha} \pi^{d / 2} \Gamma(-\alpha) / \Gamma(k+d / 2)$

$b_{k, d}=2^{-2 k+1}(-1)^{k-1} \pi^{d / 2} /\{\Gamma(k+d / 2) k !\}$

In the same paper the equation (5.8) gives the expression for the Bending Energy:

$$
J_{r+1}^{d}\left(y^{*}\right)=(2 \pi)^{2} y^{T} B y
$$

where $r=1=\alpha$.

\section{TWO DIMENSIONAL CASE}

In the two dimensional case $d=2, \alpha=1=k$ (Theorem 1 pag. 333 Kent and Mardia 1994).

$$
\begin{aligned}
\sigma_{1}(h) & =b_{k, d}|h|^{2} \log |h| \\
b_{k, d} & =b_{1,2}=2^{-1}(-1)^{0} \frac{\pi^{2 / 2}}{\Gamma\left(1+\frac{1}{2}\right) 1 !}=\frac{1}{2} \frac{\pi}{\Gamma(2)}=\frac{\pi}{2}
\end{aligned}
$$

Covariation function $\sigma(h)=\frac{\pi}{2}|h|^{2} \log |h|$ leads to Bending Energy $J=(2 \pi)^{2} y^{T} B y$.
So, with $\sigma(h)=|h|^{2} \log |h|$ we obtain a Bending Energy

$$
J=(2 \pi)^{2} y^{T} B y \times \frac{2}{\pi}=8 \pi y^{T} B y
$$

If we use $\sigma(h)=|h|^{2} \log |h|^{2}=2|h|^{2} \log |h|$ we obtain a Bending Energy

$$
J=(2 \pi)^{2} y^{T} B y \times \frac{4}{\pi}=16 \pi y^{T} B y
$$

\section{THREE DIMENSIONAL CASE}

In the three dimensional case $d=3, \alpha=1 / 2$ (Theorem 1 pag. 333 Kent and Mardia 1994).

$$
\begin{aligned}
\sigma_{1 / 2}(h) & =c_{\alpha, d}|h|^{1 / 2} \\
c_{1 / 2,3} & =2^{-1} \frac{\pi^{3 / 2} \Gamma(-1 / 2)}{\Gamma(1 / 2+3 / 2)}=\frac{1}{2} \frac{\pi^{3 / 2}(-2) \sqrt{\pi}}{\Gamma(2)}=-\pi^{2}
\end{aligned}
$$

where we used the properties $\Gamma(2)=1$ and $\Gamma(-1 / 2)=$ $-2 \sqrt{\pi}$.

So $\sigma_{1 / 2}(h)=-\pi^{2}|h|^{1 / 2}$ gives $J=(2 \pi)^{3} y^{T} B y$.

Hence $\sigma(h)=-|h|^{1 / 2}$ gives

$$
J=\frac{8 \pi^{3}}{\pi^{2}} y^{T} B y=8 \pi y^{T} B y
$$

